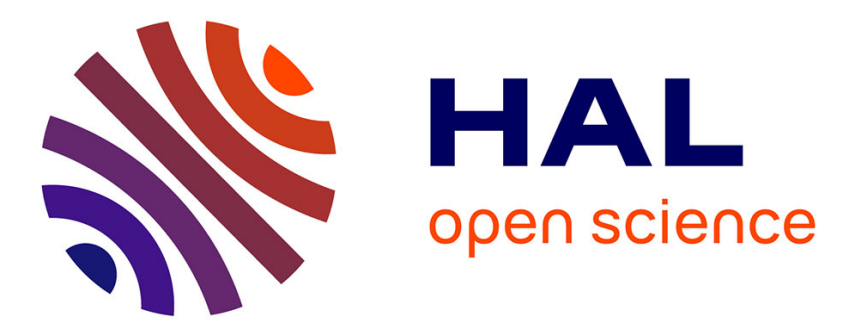

\title{
Explicit uniform estimation of rational points I. Estimation of heights
}

\author{
Huayi Chen
}

\section{To cite this version:}

Huayi Chen. Explicit uniform estimation of rational points I. Estimation of heights. 2009. hal00396374

\section{HAL Id: hal-00396374 \\ https://hal.science/hal-00396374}

Preprint submitted on 17 Jun 2009

HAL is a multi-disciplinary open access archive for the deposit and dissemination of scientific research documents, whether they are published or not. The documents may come from teaching and research institutions in France or abroad, or from public or private research centers.
L'archive ouverte pluridisciplinaire HAL, est destinée au dépôt et à la diffusion de documents scientifiques de niveau recherche, publiés ou non, émanant des établissements d'enseignement et de recherche français ou étrangers, des laboratoires publics ou privés. 


\title{
EXPLICIT UNIFORM ESTIMATION OF RATIONAL POINTS I. ESTIMATION OF HEIGHTS
}

\author{
Huayi Chen
}

\begin{abstract}
By using the slope method, we obtain an explicit uniform estimation for the density of rational points in an arithmetic projective variety with given degree and dimension, embedded in a given arithmetic projective space.
\end{abstract}

Résumé. - On obtient, en utilisant la méthode de pentes, une estimation uniforme et explicite de la densité des points rationnels dans une variété arithmétique projective de degré et dimension fixés, plongée dans un espace projectif arithmétique donné.

\section{Contents}

1. Introduction.

2. Slope inequalities.

4. Estimations of Hilbert-Samuel functions.................... 17

Appendix A. Computation of norms of linear operators.......... 23

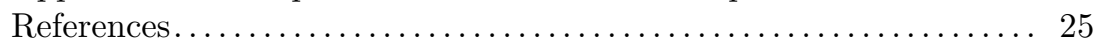

\section{Introduction}

Let $K$ be a number field and $X$ be a projective variety defined over $K$. The complexity of the rational points of $X$ is measured by the height function. Northcott's theorem asserts that there are only finitely many rational points of $X$ with bounded height. Namely, for any real number $B>0$, the set $S(X ; B):=\{P \in X(K) \mid H(P) \leqslant$ $B\}$ is finite, where $H(P)$ is the height of $P$. Let $N(X ; B)=\# S(X ; B)$ be the counting function of $X$. The asymptotic behaviour of $N(X ; B)$ when $B$ goes to infinity describes the density of $X(K)$. For example, $N(X ; B)=O(1)$ if and only if $X(K)$ is finite.

Among the estimations of the counting function $N(X ; B)$, the work of HeathBrown 31 is of a uniform nature, where the word "uniform" concerns all closed subvarieties with given degree and dimension in a projective space. His idea is to use 
a determinant argument, inspired by Bombieri and Pila [2], and Pila 39], which can be summarized as follows. The monomials of a certain degree evaluated on a family of rational points in $S(X ; B)$ having the same reduction modulo some prime number form a matrix whose determinant is zero by a local estimation. Hence there exists a hypersurface of $X$ containing all rational points in the family. The set $S(X ; B)$ is then covered by several hypersurfaces of bounded degree. The upper bound of $N(X ; B)$ is thus obtained by estimating the number of these auxiliary hypersurfaces.

The results in 31 are obtained for $K=\mathbb{Q}$. Further research in this direction includes works of Heath-Brown, Browning, Salberger and Broberg etc. (see [9, 12, 13, 14, 15, 24, 41, 42]). In particular, Broberg has generalized [31] to the number field case. It should be pointed out that the determinant argument plays an important role in most of the works cited above.

Based on an observation of Bost that the determinant argument mentioned above is quite similar to his slope method [3], or to the interpolation matrix method of Laurent 35], we revisit this problem in the context of Arakelov geometry by using the slope method. The aim of this approach is twofold. On one hand, it avoids using Siegel's lemma and hence treats the problem for all number fields in a uniform way without supplementary difficulties. On the other hand, the geometrical interpretation permits us to establish explicit estimations.

Recall the main theorem of [9] which generalizes the Theorem 14 of [31].

Theorem. - Let $X$ be an integral closed sub-variety of $\mathbb{P}_{K}^{n}, n \in \mathbb{N}_{*}$. Let $d$ and $\delta$ be respectively the dimension and the degree of $X$, and $\varepsilon>0$ be a positive number. Assume that the ideal $I \subset K\left[T_{0}, \cdots, T_{n}\right]$ of $X$ is generated by homogeneous polynomials of degrees at most $\tau$, where $\tau \in \mathbb{N}_{*}$. Then there exists an integer a depending only on $n, \tau$ and $\varepsilon$, an integer $k$ satisfying

$$
k \ll_{n, \tau, \varepsilon} B^{(d+1) / \sqrt[d]{\delta}+\varepsilon}
$$

and a family $\left(F_{i}\right)_{i=0}^{k}$ of homogeneous polynomials of degree $\leqslant$ a which are not identically zero on $X$ and such that

$$
S(X ; B) \subset \bigcup_{i=0}^{k}\left\{x \in X(K) \mid F_{i}(x)=0\right\},
$$

where $S(X ; B)$ denotes the set of all rational points of $X$ with height $\leqslant B$.

Note that Heath-Brown had considered the case where $X$ is a hypersurface (that is, the ideal $I$ is principal) defined over $\mathbb{Q}$ and Broberg has investigated the general case.

The aim of this article and the companion one $[\mathbf{1 9}$ is to remove the supplementary assumption on the degrees of polynomials generating $I$ and to calculate explicitly the constants $a$ and $k$ figuring in the above theorem. For the first purpose, we shall use the theory of Cayley-Chow form to construct explicitly a system of generators of the variety $X$. This theory, in its classical form, is due to Chow and Van de Warden 21]. Their original objective was to describe a projective variety $X$ by one single homogeneous equation, called the Chow form of $X$. Later this theory 
has been applied in transcendental number theory by Gelfond [28], Nesterenko [36], Brownawell and Waldschmidt [1], and Philippon 37]. By [36] (see also 10]), one can construct explicitly a system of generators of a projective variety from its Chow form in controlling the degrees and the heights of these polynomials. However, the degrees of the polynomials thus constructed are in general much larger than the degree of the variety. To overcome this difficulty, we shall use a variant called the Cayley form. This approach is inspired by a work of Catanese [16 (see also [27]). The Cayley form permits us to construct a system of generators of lower degree. Using the theory of Cayley form, we can remove the supplementary condition in the above theorem.

The explicit computation of the constants $a$ and $k$ requires highly non-trivial effective minoration of the arithmetic Hilbert-Samuel function developed in [22] where higher Chow forms involve, and also several new estimations in the slope theory. It is known since the article 29] of Gillet and Soulé that the coefficient of the leading term of the arithmetic Hilbert-Samuel function is equal to the normalized auto-intersection number in the sense of the arithmetic intersection theory. In order to obtain explicit lower bounds of the arithmetic Hilbert-Samuel function, we shall reformulate the result of David and Philippon in the language of slope method. Note that the lower bound thus obtained is not asymptotically optimal. The coefficient of its leading term is smaller than the normalized auto-intersection number. This result will be used to prove that all rational points of small height are contained in a single hypersurface of low degree.

We also study effective upper bounds of the arithmetic Hilbert-Samuel function (or more generally, the maximal slope variant of it) and obtain an explicit upper bound in terms of the essential minimum of the variety. The proof is based on the slope inequality applied to the evaluation map on points of small height.

It turns out that these results have their own interest in Arakelov geometry, and deserve to be written independently. In the forthcoming article [19], we shall prove the explicit version of the theorem previously cited.

This article is organized as follows. In the second section, we establish several slope inequalities and discuss their arithmetic consequences. The third section is devoted to the construction of Cayley forms and its application in the estimation of the complexity of the singular locus of an arithmetic projective variety. Finally in the fourth section, we discuss the estimation of the geometric and arithmetic HilbertSamuel functions in the framework of Arakelov geometry. Several computation of norms of tensor operators on Hermitian spaces are left in the Appendix.

We present the notation and the terminologies that we shall use in the current article and in [19].

Notation. - 1. Denote by $K$ a number field and by $\mathcal{O}_{K}$ its integer ring.

2. Any maximal ideal $\mathfrak{p}$ of $\mathcal{O}_{K}$ corresponds to a discrete valuation $v_{\mathfrak{p}}$ on $K$. Denote by $\mathbb{F}_{\mathfrak{p}}$ its residue field, by $N_{\mathfrak{p}}$ the cardinal of $\mathbb{F}_{\mathfrak{p}}$ and by $|\cdot|_{\mathfrak{p}}$ the absolute value on $K$ such that $|a|_{\mathfrak{p}}=N_{\mathfrak{p}}^{-v_{\mathfrak{p}}(a)}$ for any $a \in K^{\times}$, which extends continuously to the completion $K_{\mathfrak{p}}$ of $K$ (with respect to $v_{\mathfrak{p}}$ ). 
For any embedding $\sigma: K \rightarrow \mathbb{C}$, denote by $|\cdot|_{\sigma}$ the absolute value on $K$ such that $|a|_{\sigma}=|\sigma(a)|$, where $|\cdot|$ is the usual absolute value on $\mathbb{C}$.

3. By arithmetic projective variety we mean an integral projective $\mathcal{O}_{K}$-scheme which is flat over $\operatorname{Spec} \mathcal{O}_{K}$.

4. Let $X$ be an arithmetic projective variety. A Hermitian vector bundle on $X$ is a pair $\bar{E}=\left(E,\left(\|\cdot\|_{\sigma}\right)_{\sigma: K \rightarrow \mathbb{C}}\right)$, where $E$ is a locally free $\mathcal{O}_{X}$-module of finite rank, and for any embedding $\sigma: K \rightarrow \mathbb{C},\|\cdot\|_{\sigma}$ is a continuous Hermitian metric on $E_{\sigma}(\mathbb{C})$, invariant under the action of the complex conjugation. The rank of $\bar{E}$ is defined to be the rank of $E$, denoted by $\operatorname{rk}(E)$. A Hermitian vector bundle of rank 1 is called a Hermitian line bundle.

5. Let $\bar{E}$ be a Hermitian vector bundle on $\operatorname{Spec} \mathcal{O}_{K}$. The Arakelov degree of $\bar{E}$ is defined as ${ }^{(1)}$

$$
\widehat{\operatorname{deg}}(\bar{E}):=\log \#\left(E /\left(\mathcal{O}_{K} s_{1}+\cdots \mathcal{O}_{K} s_{r}\right)\right)-\frac{1}{2} \sum_{\sigma: K \rightarrow \mathbb{C}} \log \operatorname{det}\left(\left\langle s_{i}, s_{j}\right\rangle_{\sigma}\right),
$$

where $\left(s_{1}, \cdots, s_{r}\right) \in E^{r}$ forms a basis of $E_{K}$ over $K, r=\operatorname{rk}(E)$.

6. Let $\bar{E}$ be a non-zero Hermitian vector bundle on $\operatorname{Spec} \mathcal{O}_{K}$. The slope of $\bar{E}$ is

$$
\widehat{\mu}(\bar{E}):=\frac{1}{[K: \mathbb{Q}]} \frac{\widehat{\operatorname{deg}}(\bar{E})}{\operatorname{rk} E} .
$$

Denote by $\widehat{\mu}_{\max }(\bar{E})$ the maximal value of slopes of all non-zero Hermitian subbundles (i.e., submodule of $E$ equipped with induced metrics) of $\bar{E}$ and by $\widehat{\mu}_{\min }(\bar{E})$ the minimal value of slopes of all non-zero Hermitian quotient bundle (i.e., projective quotient module of $E$ equipped with quotient metrics) of $\bar{E}$.

7. Let $\bar{E}$ and $\bar{F}$ be two non-zero Hermitian vector bundles on $\operatorname{Spec} \mathcal{O}_{K}$, and $\phi: E_{K} \rightarrow$ $F_{K}$ be a non-zero homomorphism. The height of $\phi$ is defined as

$$
h(\phi):=\frac{1}{[K: \mathbb{Q}]}\left(\sum_{\mathfrak{p}} \log \|\phi\|_{\mathfrak{p}}+\sum_{\sigma: K \rightarrow \mathbb{C}} \log \|\phi\|_{\sigma}\right),
$$

where $\|\phi\|_{\mathfrak{p}}$ and $\|\phi\|_{\sigma}$ are respectively the norms of the linear operators $\phi_{K_{\mathfrak{p}}}$ : $E_{K_{\mathfrak{p}}} \rightarrow F_{K_{\mathfrak{p}}}$ and $\phi_{\sigma}: E_{\sigma, \mathbb{C}} \rightarrow F_{\sigma, \mathbb{C}}$.

8. For any integer $n \geqslant 1$ and any $n$-tube $\left(\bar{E}_{1}, \cdots, \bar{E}_{n}\right)$ of non-zero Hermitian vector bundles on $\operatorname{Spec} \mathcal{O}_{K}$, denote by $\varrho\left(\bar{E}_{1}, \cdots, \bar{E}_{n}\right)$ the difference

$$
\widehat{\mu}_{\max }\left(\bar{E}_{1} \otimes \cdots \otimes \bar{E}_{n}\right)-\sum_{i=1}^{n} \widehat{\mu}_{\max }\left(\bar{E}_{i}\right)
$$

For simplifying notation, we use the expression $\varrho^{(n)}(\bar{E})$ to denote $\varrho(\underbrace{\bar{E}, \cdots, \bar{E}}_{n \text { copies }})$.

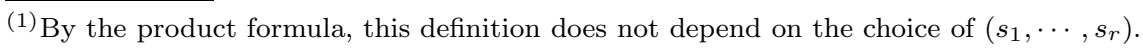




\section{Slope inequalities}

We firstly recall the basic ingredients of Bost's slope theory (for references, see [3, [5, 17, 6]), then discuss several slope (in)equalities and their arithmetic consequences.

We begin with the following classical slope (in)equalities relating the source and the target of a homomorphism between Hermitian vector bundles, see [3, Appendix A].

Proposition 2.1. - Assume that $\bar{E}$ and $\bar{F}$ are two Hermitian vector bundles on $\operatorname{Spec} \mathcal{O}_{K}$, and $\phi: E_{K} \rightarrow F_{K}$ is a non-zero $K$-linear homomorphism.

1) If $\phi$ is injective, then $\widehat{\mu}_{\max }(\bar{E}) \leqslant \widehat{\mu}_{\max }(\bar{F})+h(\phi)$.

2) If $\phi$ is surjective, then $\widehat{\mu}_{\min }(\bar{E}) \leqslant \widehat{\mu}_{\min }(\bar{F})+h(\phi)$.

3) If $\phi$ is an isomorphism, then $\widehat{\mu}(\bar{E})=\widehat{\mu}(\bar{F})+\frac{1}{\operatorname{rk}(E)} h\left(\Lambda^{\mathrm{rk}(E)} \phi\right)$.

2.1. A slope equality. - We give below a variant of the slope equality in Proposition 2.1 3) where the target Hermitian vector bundle can be written as a direct sum of Hermitian line bundles.

Proposition 2.2. - Let $\bar{E}$ be a Hermitian vector bundle of rank $r>0$ on $\operatorname{Spec} \mathcal{O}_{K}$ and $\left(\bar{L}_{i}\right)_{i \in I}$ be a family of Hermitian line bundles on $\operatorname{Spec} \mathcal{O}_{K}$. If $\phi: E_{K} \rightarrow \bigoplus_{i \in I} L_{i, K}$ is an injective homomorphism, then there exists a subset $I_{0}$ of cardinal $r$ of $I$ such that the following equality holds

$$
\widehat{\mu}(\bar{E})=\frac{1}{r}\left[\sum_{i \in I_{0}} \widehat{\mu}\left(\bar{L}_{i}\right)+h\left(\Lambda^{r}\left(\operatorname{pr}_{I_{0}} \circ \phi\right)\right)\right],
$$

where $\operatorname{pr}_{I_{0}}: \bigoplus_{i \in I} L_{i, K} \rightarrow \bigoplus_{i \in I_{0}} L_{i, K}$ is the projection.

Proof. - Since $\phi$ is injective, there exists $I_{0} \subset I$ of cardinal $r$ such that $\operatorname{pr}_{I_{0}} \circ \phi$ is an isomorphism. Therefore, Proposition 2.1 3) implies

$$
\widehat{\mu}(\bar{E})=\widehat{\mu}\left(\bigoplus_{i \in I_{0}} \bar{L}_{i}\right)+\frac{1}{r} h\left(\Lambda^{r}\left(\operatorname{pr}_{I_{0}} \circ \phi\right)\right)=\frac{1}{r}\left[\sum_{i \in I_{0}} \widehat{\mu}\left(\bar{L}_{i}\right)+h\left(\Lambda^{r}\left(\operatorname{pr}_{I_{0}} \circ \phi\right)\right)\right] .
$$

2.2. Tensor product and image. - Let $\left(\bar{E}_{i}\right)_{i=1}^{n}$ be a family of non-zero Hermitian vector bundles on $\operatorname{Spec} \mathcal{O}_{K}$, and $\bar{E}=\bigotimes_{i=1}^{n} \bar{E}_{i}$ be their tensor product. One always has

$$
\widehat{\mu}_{\max }(\bar{E}) \geqslant \sum_{i=1}^{n} \widehat{\mu}_{\max }\left(\bar{E}_{i}\right)
$$

The inverse inequality is a conjecture of Bost [顿, which is still an open problem.

If $\bar{L}$ is a Hermitian line bundle on $\operatorname{Spec} \mathcal{O}_{K}$, then for any non-zero Hermitian vector bundle $\bar{E}$ on $\operatorname{Spec} \mathcal{O}_{K}$, one has

$$
\widehat{\mu}_{\max }(\bar{E} \otimes \bar{L})=\widehat{\mu}_{\max }(\bar{E})+\widehat{\mu}(\bar{L})=\widehat{\mu}_{\max }(\bar{E})+\widehat{\mu}_{\max }(\bar{L}) .
$$


Hence $\varrho(\bar{E}, \bar{L})=\varrho(\bar{L}, \bar{E})=0$. More generally, for any family $\left(\bar{E}_{i}\right)_{i=1}^{n}$ of non-zero Hermitian vector bundles, one has $\varrho\left(\bar{E}_{1}, \bar{E}_{2}, \cdots, \bar{E}_{n}\right)=0$ if all Hermitian vector bundles except at most one among $E_{1}, \cdots, E_{n}$ are direct sum of Hermitian line bundles (see Notation 8.).

Let $\left(\bar{E}_{i}\right)_{i=1}^{n}$ and $\left(\bar{F}_{j}\right)_{j=1}^{m}$ be two families of non-zero Hermitian vector bundles on $\operatorname{Spec} \mathcal{O}_{K}$. By (2), one has

$$
\varrho\left(\bar{E}_{1}, \cdots, \bar{E}_{n}, \bar{F}_{1}, \cdots, \bar{F}_{m}\right) \geqslant \varrho\left(\bar{E}_{1}, \cdots, \bar{E}_{n}\right)+\varrho\left(\bar{F}_{1}, \cdots, \bar{F}_{m}\right) .
$$

In particular, for any non-zero Hermitian vector bundle $\bar{E}$ on $\operatorname{Spec} \mathcal{O}_{K}$, one has

$$
\forall n, m \in \mathbb{N}^{*}, \quad \varrho^{(n+m)}(\bar{E}) \geqslant \varrho^{(n)}(\bar{E})+\varrho^{(m)}(\bar{E}) .
$$

Moreover, (2) implies that $\varrho^{(m)}(\bar{E}) \geqslant 0$ for any $m \in \mathbb{N}^{*}$. Hence by (4), the sequence $\left(\varrho^{(n)}(\bar{E})\right)_{n \geqslant 1}$ is increasing.

Remark 2.3. - By the duality between maximal slope and minimal slope $\widehat{\mu}_{\min }(\bar{E})=-\widehat{\mu}_{\max }\left(\bar{E}^{\vee}\right)$, one has

$$
\varrho\left(\bar{E}_{1}^{\vee}, \cdots, \bar{E}_{n}^{\vee}\right)=\sum_{i=1}^{n} \widehat{\mu}_{\min }\left(\bar{E}_{i}\right)-\widehat{\mu}_{\min }\left(\bar{E}_{1} \otimes \cdots \otimes \bar{E}_{n}\right)
$$

Bost's conjecture can be reformulated as : $\varrho\left(\bar{E}_{1}, \cdots, \bar{E}_{n}\right) \equiv 0$ for any $n$-tube $\left(\bar{E}_{i}\right)_{i=1}^{n}$ of non-zero Hermitian vector bundles over $\operatorname{Spec} \mathcal{O}_{K}$. For estimations of $\varrho$, see [23, 8, 20]. One has

$$
\varrho\left(\bar{E}_{1}, \cdots, \bar{E}_{n}\right) \leqslant \sum_{i=2}^{n} \frac{1}{2} \log \left(\mathrm{rk} E_{i}\right) .
$$

Note that the summation index $i$ varies from 2 to $n$.

Let $\bar{E}$ and $\bar{F}$ be two Hermitian vector bundles on $\operatorname{Spec} \mathcal{O}_{K}$, and $\bar{G}$ be a Hermitian vector subbundle of $\bar{F} \otimes \bar{E}$. We call image of $\bar{G}$ in $\bar{E}$ the smallest sub- $\mathcal{O}_{K}$-module $H$ of $E$ such that $F \otimes H$ contains $G$, equipped with induced metrics. The minimal slope of $\bar{H}$ is estimated in Proposition 2.4 below, the proof of which uses the first part of Lemma A.1 in Appendix.

Proposition 2.4. - With the above notation, one has

$$
\widehat{\mu}_{\text {min }}(\bar{H}) \geqslant \widehat{\mu}_{\min }(\bar{G})-\widehat{\mu}_{\max }(\bar{F})-\varrho\left(\bar{F}, \bar{G}^{\vee}\right)-\frac{1}{2} \log (\text { rk } F) .
$$

Proof. - Let $\psi$ be the composed homomorphism $F^{\vee} \otimes G \longrightarrow F^{\vee} \otimes F \otimes E \longrightarrow E$, where the last arrow is induced by the trace homomorphism $F^{\vee} \otimes F \rightarrow \mathcal{O}_{K}$. The image of $\psi$ identifies with $H$. By Lemma A.1 1), the height of $\psi$ equals $\frac{1}{2} \log (\operatorname{rk} F)$. Thus Proposition 2.1 2) implies

$$
\widehat{\mu}_{\text {min }}(\bar{H}) \geqslant \widehat{\mu}_{\text {min }}\left(\bar{F}^{\vee} \otimes \bar{G}\right)-h(\psi)=\widehat{\mu}_{\text {min }}(\bar{G})+\widehat{\mu}_{\text {min }}\left(\bar{F}^{\vee}\right)-\varrho\left(\bar{F}, \bar{G}^{\vee}\right)-\frac{1}{2} \log (\mathrm{rk} F)
$$

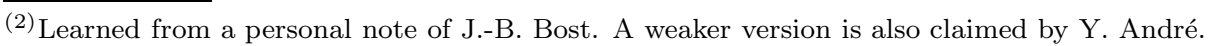


Remark 2.5. - Assume in the above proposition that $\bar{F}$ can be written as a tensor product $\bar{F}_{1} \otimes \cdots \otimes \bar{F}_{n}$. Then then same method gives the following variant of $(\mathbb{7})$ :

$$
\widehat{\mu}_{\min }(\bar{H}) \geqslant \widehat{\mu}_{\min }(\bar{G})-\sum_{i=1}^{n} \widehat{\mu}_{\max }\left(\bar{F}_{i}\right)-\varrho\left(\bar{F}_{1}, \cdots \bar{F}_{n}, \bar{G}^{\vee}\right)-\frac{1}{2} \log (\operatorname{rk} F) .
$$

2.3. Applications. - We give several applications of slope (in)equalities established in previous subsections. More applications will be discussed in $\S 3$.

In Arakelov theory, the slope inequalities are often applied on evaluation maps to obtain arithmetic results. See [6] for a survey of this method. Classically the evaluation map means the evaluation of some polynomials at one or several points in an affine space. The choice of the evaluation map is a crucial step in a typical proof of Diophantine approximation. Note that in Heath-Brown's determinant argument, there appears also this procedure. Evaluation maps in Arakelov geometry are quite similar to classical ones, but their construction is of geometrical nature. Let $X$ be a projective variety over Spec $K$ and $L$ be an ample line bundle on $X$. Let $Y$ be a closed subscheme of $X$ and $i: Y \rightarrow X$ be the inclusion morphism. The evaluation map (of global sections of $L)$ on $Y$ is the $K$-linear mapping from $H^{0}(X, L)$ to $H^{0}\left(Y, i^{*} L\right)$ defined by restriction of sections.

To apply the slope method, we also need a metric structure. Let $n \geqslant 1$ be an integer and $\overline{\mathcal{E}}$ be a Hermitian vector bundle of rank $n+1$ on $\operatorname{Spec} \mathcal{O}_{K}$. Denote by $\pi: \mathbb{P}(\mathcal{E}) \rightarrow \operatorname{Spec} \mathcal{O}_{K}$ the structural morphism. Let $\mathcal{L}:=\mathcal{O}_{\mathbb{P}(\mathcal{E})}(1)$ be the universal quotient of $\pi^{*} \mathcal{E}$. The Hermitian metrics on $\mathcal{E}$ induce by quotient a structure of Hermitian metrics on $\mathcal{L}$ which define a Hermitian line bundle $\overline{\mathcal{L}}$ on $\mathbb{P}(\mathcal{E})$. For any integer $D \geqslant 1$, let $E_{D}=H^{0}\left(\mathbb{P}(\mathcal{E}), \mathcal{L}^{\otimes D}\right)$ and let $r(D)$ be its rank over $\mathcal{O}_{K}$, which is equal to $\left(\begin{array}{c}n+D \\ D\end{array}\right)$. For any $\sigma \in \Sigma_{\infty}$, denote by $\|\cdot\|_{\sigma, \text { sup }}$ the norm on $E_{D, \sigma}:=E_{D} \otimes_{\mathcal{O}_{K}, \sigma} \mathbb{C}$ such that

$$
\forall s \in E_{D, \sigma},\|s\|_{\sigma, \sup }=\sup _{x \in \mathbb{P}\left(\mathcal{E}_{K}\right)_{\sigma}(\mathbb{C})}\|s(x)\|_{\sigma}
$$

Let $\|\cdot\|_{\sigma, J}$ be the Hermitian metric of John (cf. [32], see also [26, Definition-Theorem 2.4]) associated to the norm $\|\cdot\|_{\sigma \text {,sup }}$. Recall that, for any $s \in E_{D, \sigma}$, the following inequalities hold

$$
\|s\|_{\sigma, \mathrm{sup}} \leqslant\|s\|_{\sigma, J} \leqslant \sqrt{r(D)}\|s\|_{\sigma, \mathrm{sup}},
$$

where $r(D)$ is the rank of $E_{D}$. The $\mathcal{O}_{K}$-module $E_{D}$, equipped with Hermitian metrics $\|\cdot\|_{\sigma, J}$, forms a Hermitian vector bundle $\bar{E}_{D}$ on $\operatorname{Spec} \mathcal{O}_{K}$.

Remark 2.6. - As an $\mathcal{O}_{K}$-module, $E_{D}$ is canonically isomorphic to $S^{D} \mathcal{E}$. Thus for any $\sigma \in \Sigma_{\infty}$, the Hermitian metric on $\mathcal{E}_{\sigma, \mathbb{C}}$ induces by symmetric power a Hermitian metric $\|\cdot\|_{\sigma, \text { sym }}$ on $S^{D} \mathcal{E}_{\sigma, \mathbb{C}}$. Denote by $S^{D} \overline{\mathcal{E}}$ the corresponding Hermitian vector bundle on $\operatorname{Spec} \mathcal{O}_{K}$. Note that for any $\sigma \in \Sigma_{\infty}$, both metrics $\|\cdot\|_{\sigma, J}$ and $\|\cdot\|_{\sigma, \text { sym }}$ are invariant under the action of the unitary group $U\left(\mathcal{E}_{\sigma, \mathbb{C}},\|\cdot\|_{\sigma}\right)$, therefore they are proportional and the proportion is independent of $\sigma$. We denote by $C_{0}(D)$ the constant such that, for any $0 \neq s \in E_{D, \sigma}$,

$$
\log \|s\|_{\sigma, J}=\log \|s\|_{\sigma, \mathrm{sym}}+C_{0}(D) .
$$


Proposition 2.7. - With the above notation, the following inequality holds:

$$
0 \leqslant C_{0}(D) \leqslant \log \sqrt{r(D)}, \quad \text { where } r(D)=\operatorname{rk}\left(E_{D}\right) .
$$

Proof. - Let $s$ be a non-zero section in $H^{0}\left(X_{\sigma, \mathbb{C}}, \mathcal{L}_{\sigma, \mathbb{C}}\right)$. By definition, one has

$$
\left\|s^{D}\right\|_{\sigma, \mathrm{sup}}=\|s\|_{\sigma}^{D}=\left\|s^{D}\right\|_{\sigma, \mathrm{sym}} .
$$

As $C_{0}(D)=\log \left\|s^{D}\right\|_{\sigma, J}-\log \left\|s^{D}\right\|_{\sigma, \text { sym }}$, by (9), we obtain (11).

Remark 2.8. - By Proposition 2.1 2), Proposition 2.7 implies that

$$
\widehat{\mu}_{\min }\left(S^{D \overline{\mathcal{E}}}\right)-\frac{1}{2} \log r(D) \leqslant \widehat{\mu}_{\min }\left(\bar{E}_{D}\right) \leqslant \widehat{\mu}_{\min }\left(S^{D \overline{\mathcal{E}}}\right) .
$$

The following corollary gives an explicit lower bound of the minimal slope of $\bar{E}_{D}$ in terms of the minimal slope of $\overline{\mathcal{E}}$.

Corollary 2.9. - For any integer $D \geqslant 1$,

$$
\widehat{\mu}_{\min }\left(\bar{E}_{D}\right) \geqslant D \widehat{\mu}_{\min }(\overline{\mathcal{E}})-\varrho^{(D)}\left(\overline{\mathcal{E}}^{\vee}\right)-\frac{1}{2} D \log (n+1) .
$$

Proof. - By definition, one has $\widehat{\mu}_{\min }\left(\overline{\mathcal{E}}^{\otimes D}\right)=D \widehat{\mu}_{\min }(\overline{\mathcal{E}})-\varrho^{(D)}\left(\overline{\mathcal{E}}^{\vee}\right)$ (see (包)). Moreover, $S^{D} \overline{\mathcal{E}}$ is a quotient of $\overline{\mathcal{E}}^{\otimes D}$, so $\widehat{\mu}_{\min }\left(S^{D} \overline{\mathcal{E}}\right) \geqslant \widehat{\mu}_{\min }\left(\overline{\mathcal{E}}^{\otimes D}\right)$. Hence we obtain, according to (12),

(14) $\widehat{\mu}_{\min }\left(\bar{E}_{D}\right) \geqslant \widehat{\mu}_{\min }\left(S^{D \overline{\mathcal{E}}}\right)-\frac{1}{2} \log r(D) \geqslant D \widehat{\mu}_{\min }(\overline{\mathcal{E}})-\varrho^{(D)}\left(\overline{\mathcal{E}}^{\vee}\right)-\frac{1}{2} \log r(D)$.

Finally, (13) comes from (14) and the estimation $r(D)=\left(\begin{array}{c}n+D \\ D\end{array}\right) \leqslant(n+1)^{D}$.

Definition 2.10. - If $P$ is a rational point of $\mathbb{P}\left(\mathcal{E}_{K}\right)$, it extends in a unique way to a section $\mathcal{P}$ of $\pi$. The height of the point $P$ with respect to $\overline{\mathcal{L}}$ is by definition the slope (see Notation 6 .) of the Hermitian line bundle $\mathcal{P}^{*}(\overline{\mathcal{L}})$ on $\operatorname{Spec} \mathcal{O}_{K}$, denoted by $h_{\overline{\mathcal{L}}}(P)$.

Proposition 2.11. - Let $D \geqslant 1$ be an integer and $\bar{I}$ be a Hermitian vector subbundle of $\bar{E}_{D}$. Let $\mathcal{Y}$ be the subscheme of $\mathbb{P}(\mathcal{E})$ defined by annihilation of $I$. Suppose that $P$ is a rational point of $\mathbb{P}\left(\mathcal{E}_{K}\right)$ which is not in $\mathcal{Y}(K)$. Denote by $\mathcal{P}$ the $\mathcal{O}_{K}$-point of $\mathbb{P}(\mathcal{E})$ extending $P$. For any finite place $\mathfrak{p}$ of $K$, let $\alpha_{\mathfrak{p}}$ be as follows:

$$
\alpha_{\mathfrak{p}}= \begin{cases}1, & (\mathcal{P} \bmod \mathfrak{p}) \in \mathcal{Y}\left(\mathbb{F}_{\mathfrak{p}}\right) \\ 0, & \text { else }\end{cases}
$$

Then, for any real number $N_{0}>0$, the following inequality holds:

$$
\#\left\{\mathfrak{p} \mid \alpha_{\mathfrak{p}}=1, N_{\mathfrak{p}} \geqslant N_{0}\right\} \leqslant \frac{D h_{\overline{\mathcal{L}}}(P)-\widehat{\mu}_{\min }(\bar{I})}{\log N_{0}} .
$$


Proof. - Let $\eta: I \rightarrow \mathcal{P}^{*} \mathcal{L}^{\otimes D}$ be the homomorphism induced by the evaluation map $E_{D} \rightarrow \mathcal{P}^{*} \mathcal{L}^{\otimes D}$. Since $P \notin \mathcal{Y}(K)$, the homomorphism $\eta_{K}$ is surjective. By the slope inequality (Proposition 2.1 2)), we have

$$
D h_{\overline{\mathcal{L}}}(P) \geqslant \widehat{\mu}_{\min }(\bar{I})-h\left(\eta_{K}\right) \geqslant \widehat{\mu}_{\min }(\bar{I})+\sum_{\mathfrak{p}} \alpha_{\mathfrak{p}} \log N_{\mathfrak{p}}
$$

Therefore the inequality (15) holds.

Let $X$ be an integral closed subscheme of $\mathbb{P}\left(\mathcal{E}_{K}\right)$ and $\mathscr{X}$ be its Zariski closure in $\mathbb{P}(\mathcal{E})$. Denote by

$$
\eta_{X, D}: E_{D, K}=H^{0}\left(\mathbb{P}\left(\mathcal{E}_{K}\right), \mathcal{L}_{K}^{\otimes D}\right) \longrightarrow H^{0}\left(X,\left.\mathcal{L}\right|_{X} ^{\otimes D}\right)
$$

the evaluation map on $X$. Denote by $F_{D}$ the saturation of $\operatorname{Im}\left(\eta_{X, D}\right)$ in $H^{0}\left(\mathscr{X},\left.\mathcal{L}\right|_{\mathscr{X}} ^{\otimes D}\right)$. Note that, for sufficiently large $D$, the homomorphism $\eta_{X, D}$ is surjective, and therefore $F_{D}=H^{0}\left(\mathscr{X},\left.\mathcal{L}\right|_{\mathscr{X}} ^{\otimes D}\right)$.

The following result show that the evaluation on a collection of rational points with small heights cannot be injective. Let $Z=\left(P_{i}\right)_{i \in I}$ be a collection of distinct rational points of $X$. The evaluation map

$$
\eta_{Z, D}: H^{0}\left(\mathbb{P}\left(\mathcal{E}_{K}\right), \mathcal{L}_{K}^{\otimes D}\right) \longrightarrow \bigoplus_{i \in I} P_{i}^{*} \mathcal{L}_{K}^{\otimes D}
$$

factorizes through $\eta_{X, D}$. Denote by

$$
\phi_{Z, D}: F_{D, K} \longrightarrow \bigoplus_{i \in I} P_{i}^{*} \mathcal{L}_{K}^{\otimes D}
$$

the homomorphism such that $\phi_{Z, D} \eta_{X, D}=\eta_{Z, D}$, where $F_{D}$ is the saturation of $\operatorname{Im}\left(\eta_{X, D}\right)$ in $H^{0}\left(\mathscr{X},\left.\mathcal{L}\right|_{\mathscr{X}} ^{\otimes D}\right)$.

We equip $F_{D}$ with quotient metrics (from that of $\bar{E}_{D}$ ) so that $\bar{F}_{D}$ becomes a Hermitian vector bundle on $\operatorname{Spec} \mathcal{O}_{K}$. Note that the quantity $\widehat{\operatorname{deg}}\left(\bar{F}_{D}\right)$ is the normalized version of the " $D^{\text {th }}$-height" of $X$, defined and studied in [40, §2.2].

Proposition 2.12. - Assume that

$$
\sup _{i \in I} h_{\overline{\mathcal{L}}}\left(P_{i}\right)<\frac{\widehat{\mu}\left(\bar{F}_{D}\right)}{D}-\frac{1}{2 D} \log r_{1}(D), \quad \text { where } r_{1}(D)=\operatorname{rk}\left(F_{D}\right) .
$$

Then the homomorphism $\phi_{Z, D}$ cannot be injective.

Proof. - Assume that $\phi_{Z, D}$ is injective. By Proposition 2.2, there exists $I_{0} \subset I$ of cardinal $r_{1}(D)$ such that

$$
\widehat{\mu}\left(\bar{F}_{D}\right)=\frac{1}{r_{1}(D)}\left[\sum_{i \in I_{0}} D h_{\overline{\mathcal{L}}}\left(P_{i}\right)+h\left(\Lambda^{r_{1}(D)}\left(\operatorname{pr}_{I_{0}} \circ \phi\right)\right)\right],
$$

The quantity $\frac{1}{r_{1}(D)} \sum_{i \in I_{0}} h_{\overline{\mathcal{L}}}\left(P_{i}\right)$ is bounded from above $\sup _{i \in I} h_{\overline{\mathcal{L}}}\left(P_{i}\right)$. Furthermore, one has

$$
h\left(\Lambda^{r_{1}(D)}\left(\operatorname{pr}_{I_{0}} \circ \phi_{Z, D}\right)\right) \leqslant r_{1}(D) h\left(\operatorname{pr}_{I_{0}} \circ \phi_{Z, D}\right) \leqslant \frac{1}{2} r_{1}(D) \log r_{1}(D) .
$$


Hence

$$
\max _{i \in I} h_{\overline{\mathcal{L}}}\left(P_{i}\right) \geqslant \frac{\widehat{\mu}\left(\bar{F}_{D}\right)}{D}-\frac{1}{2 D} \log r_{1}(D) .
$$

\section{The complicity of singular locus}

In this section, we consider the following problem. Given a closed subvariety $X$ of a projective space $\mathbb{P}^{n}$, how to describe the complexity of the singular locus of $X$ by the arithmetic invariants of $X$ ? When $X$ is a hypersurface in $\mathbb{P}^{n}$ defined by a homogeneous polynomial $F\left(T_{0}, \cdots, T_{n}\right)$ of degree $d$, then the singular locus of $X$ is determined by the equations

$$
F=\frac{\partial}{\partial T_{0}} F=\cdots=\frac{\partial}{\partial T_{n}} F=0 .
$$

Therefore the ideal of $\operatorname{Sing}(X)$ is generated by $n+2$ polynomials of height $\leqslant d h(F)$. In the general case, the singular locus can be described by using the Jacobian criterion, provided a system of generators of the ideal of $X$.

Given a subvariety $X \subset \mathbb{P}^{n}$ of dimension $d$ and of degree $\delta$, a method to construct explicitly a system of polynomials defining $X$ is to use the Chow form. The Chow form $\Phi_{X}$ of $X$ is a multi-homogeneous polynomial of multi-degree $(\delta, \cdots, \delta)$ on the multi-projective space $\left(\mathbb{P}^{n}\right)^{d+1}$. The general theory of Chow and Van de Warden [21] asserts that set-theoretically any subvariety of dimension $d$ and of degree $\delta$ of $\mathbb{P}^{n}$ is uniquely determined by its Chow form.

Philippon 37 has defined the height of an arithmetic variety as that of its Chow form and applied his height theory on criteria of algebraic independence. The Philippon height can be compared to the Arakelov height [44, 38, 7]. As mentioned in Introduction, one can construct explicitly a system of generators of a projective variety from its Chow form. This permits us in principle to understand the complexity of the singular locus of the projective variety by using the Jacobian criterion. However, the generating system obtained by the Chow form is not saturated with respect to the maximal homogeneous ideal. For example, if the projective variety is a hypersurface in a projective space defined by a homogeneous equation $F$ of degree $\delta$, then the generating system obtained from the Chow form will be the linear space of equations of the form $F G$, where $G$ runs over all homogeneous polynomials of degree $\delta d$. Therefore, if we try to estimate the complexity of the singular locus of the variety by using this linear system, supplementary errors will occur in the procedure of differential and also in that of taking determinant.

In this article, we shall use the point of view of Cayley form. This approach is inspired by [27, 16]. The construction of Cayley form is quite similar to that of Chow form. The only difference is that, in the construction of Chow form, we use Stiefel coordinates; while in that of Cayley form, we use Plücker coordinates.

In the following, we recall the definition of Chow form and Cayley form, the calculation of their heights, and the estimation of the complexity of the singular locus of a variety by using its Cayley form. In the rest of this section, let $n \in \mathbb{N}_{*}$ 
and $\overline{\mathcal{E}}$ be a Hermitian vector bundle of rank $n+1$. Denote by $\overline{\mathcal{L}}$ the invertible sheaf $\mathcal{O}_{\mathbb{P}(\mathcal{E})}(1)$ equipped with the Fubini-Study metrics. By sub-variety of $\mathbb{P}\left(\mathcal{E}_{K}\right)$ we mean a closed integral subscheme of $\mathbb{P}\left(\mathcal{E}_{K}\right)$.

3.1. Chow form and Cayley form. - Let $W$ be the product $\mathbb{P}\left(\mathcal{E}_{K}\right) \times \mathbb{P}\left(\mathcal{E}_{K}^{\vee}\right)^{d+1}$ and $\Gamma$ be the incident subvariety of $W$ which classifies all points $\left(\xi, u_{0}, \cdots, u_{d}\right)$ such that $\xi\left(u_{0}\right)=\cdots=\xi\left(u_{d}\right)=0$. Denote by $p: W \rightarrow \mathbb{P}\left(\mathcal{E}_{K}\right)$ and $q: W \rightarrow \mathbb{P}\left(\mathcal{E}_{K}^{\vee}\right)^{d+1}$ the two projections.

Proposition 3.1. - Let $X \subset \mathbb{P}\left(\mathcal{E}_{K}\right)$ be a subvariety. Then the set-theoretical intersection $\Gamma \cap p^{-1}(X)$ is irreducible. Furthermore, if we consider $\Gamma \cap p^{-1}(X)$ as a reduced subvariety of $W$, then the scheme-theoretical image $q\left(\Gamma \cap p^{-1}(X)\right)$ is a hypersurface of multi-degree $(\delta, \cdots, \delta)$.

Remark 3.2. - See [37] for an algebraic proof of this result in its generalized form, see $[7, \S 4.3]$ for a geometric proof.

The hypersurface in Proposition 3.1 corresponds to a subspace of rank one of $S^{\delta}\left(\mathcal{E}_{K}^{\vee}\right)^{\otimes(d+1)}$ whose saturation in $S^{\delta}\left(\mathcal{E}^{\vee}\right)^{\otimes(d+1)}$ determines a Hermitian line subbundle $\bar{\Phi}_{X}$ of $S^{\delta}\left(\overline{\mathcal{E}}^{\vee}\right)^{\otimes(d+1)}$. The generic fibre $\Phi_{X, K}$ is called the Chow form of $X$. The Philippon height of the arithmetic variety $X$ is defined as $h_{\mathrm{Ph}}(X):=-\widehat{\mu}\left(\bar{\Phi}_{X}\right)$.

Let $s^{(i)}: \mathcal{E}_{K}^{\vee} \rightarrow \mathcal{E}_{K}(0 \leqslant i \leqslant d)$ be generic antisymmetric homomorphisms and $\xi$ be a generic element in $\mathcal{E}_{K}^{\vee}$. The expression $\Phi_{X, K}\left(s^{(0)} \xi, \cdots, s^{(d)} \xi\right)$ represents a multihomogeneous polynomial of degree $\delta$ in each $s^{(i)}$ and of degree $(d+1) \delta$ in $\xi$. By specifying $s^{(i)}$, one obtains a linear system $J_{X, K}$ of homogeneous polynomials of degree $(d+1) \delta$ on $\mathcal{E}_{K}^{\vee}$. The heights of these equations can be estimated by the Philippon height of $X$. The linear system $J_{X, K}$ defines a subscheme $\widetilde{X}$ of $\mathbb{P}\left(\mathcal{E}_{K}\right)$ containing $X$. By 36, Lemma 11], it coincides with $X$ on an open subscheme containing the regular subscheme $X_{\text {reg. }}$. Furthermore, $\widetilde{X}_{\text {red }}=X$. However, in general the homogeneous ideal generated by $J_{X, K}$ is not saturated with respect to the maximal homogeneous ideal $\bigoplus_{n \geqslant 1} S^{n} \mathcal{E}_{K}$. For example, in the case where $X$ is a hypersurface of degree $\delta$ defined by a homogeneous polynomial $F$, one has $J_{X, K}=F \cdot S^{\delta d} \mathcal{E}_{K}$.

In the following, we introduce a variant of the Chow form, called the Cayley form of $X$. The advantage of the Cayley form is that we can construct from it a system of generators of $X$ which are of degree $\delta$.

Recall that in the construction of the Chow form, one has actually used the Stiefel coordinates of the Grassmannian. If we use Plüker coordinates instead, the same procedure leads to the so-called Cayley form. Let $\check{G}=\operatorname{Gr}\left(d+1, \mathcal{E}_{K}^{\vee}\right)$ be the Grassmannian which classifies all quotients of rank $d+1$ of $\mathcal{E}_{K}^{\vee}$ (or equivalently, all subspaces of rank $d+1$ of $\mathcal{E}_{K}$ ). Denote by $\Gamma^{\prime}$ the incident subvariety of $\mathbb{P}\left(\mathcal{E}_{K}\right) \times \check{G}$ which classifies all points $(\xi, U)$ such that $\xi(U)=0$ (here we consider $U$ as a subspace of $\left.\mathcal{E}_{K}\right)$. Let $p^{\prime}: \mathbb{P}\left(\mathcal{E}_{K}\right) \times \check{G} \rightarrow \mathbb{P}\left(\mathcal{E}_{K}\right)$ and $q^{\prime}: \mathbb{P}\left(\mathcal{E}_{K}\right) \times \check{G} \rightarrow \breve{G}$ be the two projections.

Proposition 3.3 (see 27 §3.2.B). - Let $X \subset \mathbb{P}\left(\mathcal{E}_{K}\right)$ be a subvariety of dimension $d$ and of degree $\delta$. The set-theoretical intersection $\Gamma^{\prime} \cap p^{\prime-1}(X)$ is irreducible. 
Furthermore, if we consider $\Gamma^{\prime} \cap p^{\prime-1}(X)$ as a reduced subvariety of $W^{\prime}$, the schemetheoretical image $q^{\prime}\left(\Gamma^{\prime} \cap p^{\prime-1}(X)\right)$ is a hypersurface of degree $\delta$ of $\check{G}$.

Proof. - The incident variety $\Gamma^{\prime}$ is a fibration on $\mathbb{P}\left(\mathcal{E}_{K}\right)$ in Grassmannian varieties. Since $X$ is irreducible, also is $\Gamma^{\prime} \cap p^{\prime-1}(X)=\left.p^{\prime}\right|_{\Gamma^{\prime}} ^{-1}(X)$. Denote by $Y=\Gamma^{\prime} \cap p^{\prime-1}(X)$, considered as a subvariety of $\Gamma^{\prime}$. The projection $q^{\prime}$ being proper, the image $Z=q^{\prime}(Y)$ is a closed integral subscheme of $\breve{G}$. Let $\xi=\operatorname{Spec} K^{\prime}$ be a geometric generic point of $Z$, which corresponds to a subspace $V$ of rank $d+1$ of $\mathcal{E}_{K^{\prime}}$. The fibre $Y_{\xi}$ coincides with the subscheme of $X_{K^{\prime}}$ defined by vanishing on $V$. Note that the dimension of $X_{K^{\prime}}$ is $d$. So $q^{\prime}$ maps $Y$ birationally to $Z$ and hence $\operatorname{dim} Z=\operatorname{dim} Y=\operatorname{dim} \check{G}-1$.

To calculate the degree of $Z$ in $\check{G}$, we consider the following equality of cycle classes

$$
[Z]=\left(\left.q^{\prime}\right|_{\Gamma^{\prime}}\right)_{*}\left(\left.p^{\prime}\right|_{\Gamma^{\prime}}\right)^{*}[X]=\delta\left(\left.q^{\prime}\right|_{\Gamma^{\prime}}\right)_{*}\left(\left.p^{\prime}\right|_{\Gamma^{\prime}}\right)^{*}[U]
$$

where $U$ is the projective space associated to an arbitrary quotient space of rank $d+1$ of $\mathcal{E}_{K}$. Note that $\left(\left.q^{\prime}\right|_{\Gamma^{\prime}}\right)_{*}\left(\left.p^{\prime}\right|_{\Gamma^{\prime}}\right)^{*}[U]$ is just the first Schubert class in the Grassmannian $\check{G}$ (see $[25, \S 14.7]$ ). Therefore the degree of $Z$ is $\delta$.

By Plücker's morphism $\check{G} \rightarrow \mathbb{P}\left(\Lambda^{d+1} \mathcal{E}_{K}^{\vee}\right)$, the coordinate algebra $B(\check{G})=$ $\bigoplus_{D \geqslant 0} B_{D}(\check{G})$ of $\check{G}$ is a homogeneous quotient algebra of $\bigoplus_{D \geqslant 0} S^{D}\left(\Lambda^{d+1} \mathcal{E}_{K}^{\vee}\right)$. To explain the role played by the Plücker coordinates, we consider the following construction. Denote by $\theta: \mathcal{E}_{K}^{\vee} \otimes\left(\Lambda^{d+1} \mathcal{E}_{K}\right) \rightarrow \Lambda^{d} \mathcal{E}_{K}$ the substraction homomorphism which sends $\xi \otimes\left(x_{0} \wedge \cdots \wedge x_{d}\right)$ to

$$
\sum_{i=0}^{d}(-1)^{i} \xi\left(x_{i}\right) x_{0} \wedge \cdots \wedge x_{i-1} \wedge x_{i+1} \wedge \cdots \wedge x_{d} .
$$

Let $\widetilde{\Gamma}$ be the subvariety of $\mathbb{P}\left(\mathcal{E}_{K}\right) \times \mathbb{P}\left(\Lambda^{d+1} \mathcal{E}_{K}^{\vee}\right)$ which classifies the points $(\xi, \alpha)$ such that $\theta(\xi \otimes \alpha)=0$. Let $\widetilde{p}: \mathbb{P}\left(\mathcal{E}_{K}\right) \times \mathbb{P}\left(\Lambda^{d+1} \mathcal{E}_{K}^{\vee}\right) \rightarrow \mathbb{P}\left(\mathcal{E}_{K}\right)$ and $\widetilde{q}: \mathbb{P}\left(\mathcal{E}_{K}\right) \times \mathbb{P}\left(\Lambda^{d+1} \mathcal{E}_{K}^{\vee}\right) \rightarrow$ $\mathbb{P}\left(\Lambda^{d+1} \mathcal{E}_{K}^{\vee}\right)$ be the two projections.

Proposition 3.4. - Let $X \subset \mathbb{P}\left(\mathcal{E}_{K}\right)$ be a subvariety of dimension d and of degree $\delta$. The set-theoretical intersection $\widetilde{\Gamma} \cap \widetilde{p}^{-1}(X)$ is irreducible. Moreover, if we consider $\widetilde{\Gamma} \cap \widetilde{p}^{-1}(X)$ as a reduced subvariety of $\mathbb{P}\left(\mathcal{E}_{K}\right) \times \mathbb{P}\left(\Lambda^{d+1} \mathcal{E}_{K}^{\vee}\right)$, then the scheme-theoretical image $\widetilde{q}\left(\widetilde{\Gamma} \cap \widetilde{p}^{-1}(X)\right)$ is a hypersurface of degree $\delta$ of $\mathbb{P}\left(\Lambda^{d+1} \mathcal{E}_{K}^{\vee}\right)$.

Denote by $\Psi_{X, K}$ the one-dimensional subspace of $S^{\delta}\left(\Lambda^{d+1} \mathcal{E}_{K}^{\vee}\right)$ which defines the hypersurface in Proposition 3.4. We call it the Cayley form of $X$. The saturation of $\Psi_{X, K}$ in $S^{\delta}\left(\Lambda^{d+1} \overline{\mathcal{E}}\right)$, quipped with induces metrics, is called the Cayley form of $X$. Note that the incident variety $\Gamma^{\prime}$ of $\mathbb{P}\left(\mathcal{E}_{K}\right) \times \check{G}$ is just the intersection of $\widetilde{\Gamma}$ with $\mathbb{P}\left(\mathcal{E}_{K}\right) \times \check{G}$ (embedded in $\mathbb{P}\left(\mathcal{E}_{K}\right) \times \mathbb{P}\left(\Lambda^{d+1} \mathcal{E}_{K}^{\vee}\right)$ via the Plücker morphism).

The relationship between the Plücker and the Stiefel coordinates (see [27, p.101] for details) leads to the following observation. Let $\psi_{X}$ be a representing element of $\Psi_{X, K}$, considered as a homogeneous polynomial of degree $\delta$ on $\Lambda^{d+1} \mathcal{E}_{K}^{\vee}$. Then the multihomogeneous polynomial $\phi_{X}$ of multidegree $(\underbrace{\delta, \cdots, \delta}_{d+1 \text { copies }})$ defined as

$$
\phi_{X}\left(x_{0}, \cdots, x_{d}\right):=\psi_{X}\left(x_{0} \wedge \cdots \wedge x_{d}\right)
$$


represents the Chow form $\Phi_{X, K}$ of $X$. This phenomenon can also be interpreted in the following way. Let $\tau_{\delta}: S^{\delta}\left(\Lambda^{d+1} \mathcal{E}_{K}^{\vee}\right) \rightarrow S^{\delta}\left(\mathcal{E}_{K}^{\vee}\right)^{\otimes(d+1)}$ be the homomorphism which sends $\prod_{i=1}^{\delta} x_{i, 0} \wedge \cdots \wedge x_{i, d}$ to

$$
\left(\frac{1}{(d+1) !}\right)^{\delta} \sum_{\left(\sigma_{1}, \cdots, \sigma_{\delta}\right) \in \mathfrak{S}_{d+1}^{\delta}} \operatorname{sgn}\left(\sigma_{1}\right) \cdots \operatorname{sgn}\left(\sigma_{\delta}\right) \bigotimes_{j=0}^{d} x_{1, \sigma_{1}(j)} \cdots x_{\delta, \sigma_{\delta}(j)} .
$$

Then $\Phi_{X, K}$ is just the image of $\Psi_{X, K}$ under the homomorphism $\tau$. Note that the direct sum of $\tau_{\delta}$ is a homomorphism of algebras

$$
\tau: \bigoplus_{\delta \in \mathbb{N}} S^{\delta}\left(\Lambda^{d+1} \mathcal{E}_{K}^{\vee}\right) \longrightarrow \bigoplus_{\delta \in \mathbb{N}} S^{\delta}\left(\mathcal{E}_{K}^{\vee}\right)^{\otimes(d+1)},
$$

which corresponds to the natural rational morphism $\mathbb{P}\left(\mathcal{E}_{K}^{\vee}\right)^{d+1} \rightarrow \mathbb{P}\left(\Lambda^{d+1} \mathcal{E}_{K}^{\vee}\right)$ which sends a general point $\left(x_{0}, \cdots, x_{d}\right) \in \mathcal{E}_{K}^{(d+1)}$ to $x_{0} \wedge \cdots \wedge x_{d}$. From the construction above, we obtain the following relation on the slopes of the Chow form and the Cayley form.

Corollary 3.5. - One has

$$
\widehat{\mu}\left(\bar{\Psi}_{X}\right)=\widehat{\mu}\left(\bar{\Phi}_{X}\right)+\frac{\delta}{2} \log (d+1) ! .
$$

We construct a system of generators of $X$ from $\Psi_{X, K}$. Choose a representative element $\psi_{X}$ in $\Psi_{X, K}$ and consider it as a homogeneous polynomial of degree $\delta$ on $\Lambda^{d+1} \mathcal{E}_{K}^{\vee}$. Let $x, y_{0}, \cdots, y_{d}$ be generic elements in $\mathcal{E}_{K}$ and let $\xi$ be a generic element in $\mathcal{E}_{K}^{\vee}$. For any $i=0,1 \cdots, d$, let $z_{i}=\xi(x) y_{i}-\xi\left(y_{i}\right) x$. As

$$
\begin{aligned}
& z_{0} \wedge \cdots \wedge z_{d} \\
= & \xi(x)^{d+1} y_{0} \wedge \cdots \wedge y_{d}-\sum_{i=0}^{d} \xi(x)^{d} \xi\left(y_{i}\right) y_{0} \wedge \cdots \wedge y_{i-1} \wedge x \wedge y_{i+1} \wedge \cdots \wedge y_{d} \\
= & \xi(x)^{d}\left(\xi(x) y_{0} \wedge \cdots \wedge y_{d}-\sum_{i=0}^{d} \xi\left(y_{i}\right) y_{0} \wedge \cdots \wedge y_{i-1} \wedge x \wedge y_{i+1} \wedge \cdots \wedge y_{d}\right),
\end{aligned}
$$

we obtain

$$
\begin{aligned}
\psi_{X, K}\left(z_{0} \wedge \cdots \wedge z_{d}\right)= & \xi(x)^{\delta d} \psi_{X, K}\left(\xi(x) y_{0} \wedge \cdots \wedge y_{d}\right. \\
& \left.-\sum_{i=0}^{d} \xi\left(y_{i}\right) y_{0} \wedge \cdots \wedge y_{i-1} \wedge x \wedge y_{i+1} \wedge \cdots \wedge y_{d}\right) .
\end{aligned}
$$

By specifying $x, y_{0}, \cdots, y_{d}$ in

$$
\psi_{X, K}\left(\xi(x) y_{0} \wedge \cdots \wedge y_{d}-\sum_{i=0}^{d} \xi\left(y_{i}\right) y_{0} \wedge \cdots \wedge y_{i-1} \wedge x \wedge y_{i+1} \wedge \cdots \wedge y_{d}\right)
$$

we obtain a linear system $I_{X, K}$ of polynomials of degree $\delta$ on $\mathcal{E}_{K}^{\vee}$, which also defines the subscheme $\widetilde{X}$ of $\mathbb{P}\left(\mathcal{E}_{K}\right)$. In fact, a generic antisymmetic homomorphism $\mathcal{E}_{K}^{\vee} \rightarrow \mathcal{E}_{K}$ 
acting on a generic element $\xi$ in $\mathcal{E}_{K}^{\vee}$ can be written as a linear combination over $K$ of elements of the form $\xi(x) y-\xi(y) x$, where $x$ and $y$ are generic elements in $\mathcal{E}_{K}$.

Let $\bar{I}_{X}$ be the saturated Hermitian vector subbundle of $S^{\delta} \overline{\mathcal{E}}$ whose generic fibre coincides with $I_{X, K}$. We are interested in estimating the complexity of $\bar{I}_{X}$, for which we need the following tensoriel construction of $I_{X}$.

Consider the $\mathcal{O}_{K}$-linear homomorphism $\mathcal{E} \otimes \mathcal{E}^{\otimes(d+1)} \otimes \mathcal{E}^{\vee} \rightarrow \Lambda^{d+1} \mathcal{E}$ sending $x \otimes$ $y_{0} \otimes \cdots \otimes y_{d} \otimes \xi$ to

$$
\xi(x) y_{0} \wedge \cdots \wedge y_{d}-\sum_{i=0}^{d} \xi\left(y_{i}\right) y_{0} \wedge \cdots \wedge y_{i-1} \wedge x \wedge y_{i+1} \wedge \cdots \wedge y_{d},
$$

which induces a homomorphism

$$
\Gamma^{\delta}(\mathcal{E}) \otimes \Gamma^{\delta}(\mathcal{E})^{\otimes(d+1)} \otimes \Gamma^{\delta}\left(\mathcal{E}^{\vee}\right) \rightarrow \Gamma^{\delta}\left(\Lambda^{d+1} \mathcal{E}\right),
$$

where for any projective $\mathcal{O}_{K}$-module of finite type $F, \Gamma^{\delta}(F)$ is the sub- $\mathcal{O}_{K}$-module of $F^{\otimes \delta}$ consisting of all elements which are invariant by the action of the symmetric group $\mathfrak{S}_{d}$. By the canonical isomorphism $\Gamma^{\delta}(F)^{\vee} \cong S^{\delta}\left(F^{\vee}\right)$, we obtain from (19) a homomorphism

$$
S^{\delta}\left(\Lambda^{d+1} \mathcal{E}^{\vee}\right) \rightarrow S^{\delta}\left(\mathcal{E}^{\vee}\right) \otimes S^{\delta}\left(\mathcal{E}^{\vee}\right)^{\otimes(d+1)} \otimes S^{\delta}(\mathcal{E})
$$

by duality. Denote by $f_{X}$ the composed homomorphism

$$
\Psi_{X} \longrightarrow S^{\delta}\left(\Lambda^{d+1} \mathcal{E}^{\vee}\right) \longrightarrow S^{\delta}\left(\mathcal{E}^{\vee}\right) \otimes S^{\delta}\left(\mathcal{E}^{\vee}\right)^{\otimes(d+1)} \otimes S^{\delta}(\mathcal{E})
$$

where $\Psi_{X}$ is the submodule of $S^{\delta}\left(\Lambda^{d+1} \mathcal{E}^{\vee}\right)$ corresponding to the Cayley form. Then $\bar{I}_{X}$ is just the saturation of the image (see page 6 for definition) of $\overline{f_{X}\left(\Psi_{X}\right)}$ (with induced metrics) in $S^{\delta}(\overline{\mathcal{E}})$.

Proposition 3.6. — With the above notation, the following inequality holds:

$$
\widehat{\mu}_{\min }\left(\bar{I}_{X}\right) \geqslant-h_{\mathrm{Ph}}(X)-C_{1},
$$

where the constant $C_{1}=C_{1}(\overline{\mathcal{E}}, d, \delta)$ is defined as

$$
\begin{aligned}
C_{1}= & (d+2) \widehat{\mu}_{\max }\left(S^{\delta}\left(\overline{\mathcal{E}}^{\vee}\right)\right)+\frac{1}{2}(d+2) \log \operatorname{rk}\left(S^{\delta} \mathcal{E}\right)+\varrho^{(d+2)}\left(\Gamma^{\delta}(\overline{\mathcal{E}})\right) \\
& +\frac{\delta}{2} \log ((d+2)(n-d)) .
\end{aligned}
$$

Proof. - By Proposition 2.1 and Lemma A.1 2), the slope of $\overline{f_{X}\left(\widetilde{v}\left(\Phi_{X}\right)\right)}$ can be estimated as follows:

$$
\widehat{\mu}\left(\overline{f_{X}\left(\Psi_{X}\right)}\right) \geqslant \widehat{\mu}\left(\bar{\Psi}_{X}\right)-h\left(f_{X}\right) \geqslant \widehat{\mu}\left(\bar{\Psi}_{X}\right)-\frac{\delta}{2} \log ((d+2) ! \cdot(n-d)) .
$$

By (18), this implies

$$
\widehat{\mu}\left(\overline{f_{X}\left(\Psi_{X}\right)}\right) \geqslant-h_{\mathrm{Ph}}(X)-\frac{\delta}{2} \log ((d+2)(n-d)),
$$


where we reminder that the Philippon height $h_{\mathrm{Ph}}(X)$ is defined as $-\widehat{\mu}\left(\bar{\Phi}_{X}\right)$. By Proposition 2.4 (see also Remark 2.5), we obtain

$$
\begin{aligned}
\widehat{\mu}_{\min }\left(\bar{I}_{X}\right) \geqslant \widehat{\mu}\left(\overline{f_{X}\left(\Psi_{X}\right)}\right)-(d+2) \widehat{\mu}_{\max }\left(S^{\delta}\left(\overline{\mathcal{E}}^{\vee}\right)\right) \\
-\varrho^{(d+2)}\left(\Gamma^{\delta}(\overline{\mathcal{E}})\right)-\frac{1}{2}(d+2) \log \operatorname{rk}\left(S^{\delta} \mathcal{E}\right) .
\end{aligned}
$$

Combining (22) with (23), one obtains (20).

Remark 3.7. - By [5] (see also [30, 26]), one obtains $\widehat{\mu}_{\max }\left(S^{\delta}\left(\overline{\mathcal{E}}^{\vee}\right)\right) \ll_{\overline{\mathcal{E}}} \delta$. Furthermore, one has $\varrho^{(d+2)}\left(\Gamma^{\delta}(\overline{\mathcal{E}})\right) \ll \log \operatorname{rk}\left(S^{\delta} \mathcal{E}\right) \leqslant \delta \log (n+1)$. Therefore, $C_{1} \ll \overline{\mathcal{E}, d} \delta$.

3.2. Complexity of the singular locus. - In the previous subsection, we have constructed explictely a linear systeme $I_{X, K}$ which defines a subscheme $\widetilde{X}$ of $\mathbb{P}\left(\mathcal{E}_{K}\right)$ containing $X$. Since $\widetilde{X}_{\text {red }}=X$ (see [36, ??]), we obtain $\widetilde{X}_{\text {reg }} \subset X_{\text {reg, }}$, where $\widetilde{X}_{\text {reg }}$ and $X_{\text {reg }}$ are respectively the open subschemes of all regular points of $\widetilde{X}$ and of $X$. Moreover, since $\widetilde{X}$ coincides with $X$ on a dense open subset (loc. cit.), $\widetilde{X}_{\text {reg }}$ is a dense open subscheme of $X$. Using Jacobian criterion, we shall construct from $I_{X, K}$ a linear system defining the singular locus $\widetilde{X}_{\text {sing }}$ of $\widetilde{X}$, which contains $X_{\text {sing }}$, the singular locus of $X$. Before discussing the complexity of $\widetilde{X}$, we treat a slightly general case where we consider a Hermitian subbundle of certain symmetric power of $\overline{\mathcal{E}}$ and estimate the complexity of linear systems constructed from minors of its Jacobian matrix.

For any integer $a \geqslant 1$, denote by $D_{a}: S^{a} \mathcal{E} \rightarrow \mathcal{E} \otimes S^{a-1} \mathcal{E}$ the homomorphism of derivation which sends $x_{1} \cdots x_{a}$ to $\sum_{i=1}^{a} x_{i} \otimes\left(x_{1} \cdots x_{i-1} x_{i+1} \cdots x_{a}\right)$. Suppose that $\bar{I}$ is a Hermitian subbundle of $S^{a} \overline{\mathcal{E}}$ and that $r$ is an integer such that $r \geqslant 1$. We denote by $g_{I}^{(r)}$ the following composed homomorphism

$$
I^{\otimes r} \longrightarrow S^{a}(\mathcal{E})^{\otimes r} \stackrel{D_{a}^{\otimes r}}{\longrightarrow} \mathcal{E}^{\otimes r} \otimes S^{a-1}(\mathcal{E})^{\otimes r} \longrightarrow \Lambda^{r} \mathcal{E} \otimes S^{(a-1) r}(\mathcal{E}),
$$

where the last arrow is induced by canonical homomorphisms $\mathcal{E}^{\otimes r} \rightarrow \Lambda^{r} \mathcal{E}$ and $S^{a-1}(\mathcal{E})^{\otimes r} \rightarrow S^{(a-1) r} \mathcal{E}$. Let $\bar{F}_{I}^{(r)}$ be the image of $g_{I}^{(r)}$, equipped with induced metrics. Denote by $\bar{I}^{(r)}$ the image of $\bar{F}_{I}^{(r)}$ in $S^{(a-1) r} \overline{\mathcal{E}}$.

Theorem 3.8. - With the above notation, the following inequality holds:

$$
\widehat{\mu}_{\min }\left(\bar{I}^{(r)}\right) \geqslant r \widehat{\mu}_{\min }(\bar{I})-C_{2},
$$

where the constant $C_{2}=C_{2}(\overline{\mathcal{E}}, r, a)$ is defined as

$$
\widehat{\mu}_{\max }\left(\Lambda^{r} \overline{\mathcal{E}}\right)+\frac{r}{2} \log \operatorname{rk}\left(S^{a} \mathcal{E}\right)+\log \operatorname{rk}\left(\Lambda^{r} \mathcal{E}\right)+\log \sqrt{r !}+r \log a .
$$

Proof. - By Lemma A.1 3) and 4), the height of $g_{I}^{(r)}$ is bounded from above by $\log \sqrt{r !}+r \log a$. Therefore, Proposition 2.1 2) shows that

(26) $\widehat{\mu}_{\min }\left(\bar{F}_{I}^{(r)}\right) \geqslant \widehat{\mu}_{\min }\left(\bar{I}^{\otimes r}\right)-\log \sqrt{r !}-r \log a=r \widehat{\mu}_{\min }(\bar{I})-\varrho^{(r)}\left(\bar{I}^{\vee}\right)-\log \sqrt{r !}-r \log a$ 
where $\bar{F}_{I}^{(r)}$ is the image of $g_{I}^{(r)}$, equipped with induced metrics. Note that (see $(6)$ )

$$
\varrho^{(r)}\left(\bar{I}^{\vee}\right) \leqslant \frac{r}{2} \log (\operatorname{rk} I) \leqslant \frac{r}{2} \log \left(\operatorname{rk} S^{a} \mathcal{E}\right) .
$$

By Proposition 2.4, one has

$$
\widehat{\mu}_{\min }\left(\bar{I}^{(r)}\right) \geqslant \widehat{\mu}_{\min }\left(\bar{F}_{I}^{(r)}\right)-\widehat{\mu}_{\max }\left(\Lambda^{r} \overline{\mathcal{E}}\right)-\varrho\left(\Lambda^{r} \overline{\mathcal{E}}^{\vee}, \bar{F}_{I}^{(r)}\right)-\frac{1}{2} \log \left(\operatorname{rk} \Lambda^{r} \mathcal{E}\right) .
$$

Therefore the required estimation follows from (26), 27) and the inequality

$$
\varrho\left(\Lambda^{r} \overline{\mathcal{E}}^{\vee}, \bar{F}_{I}^{(r)}\right) \leqslant \frac{1}{2} \log \left(\operatorname{rk} \Lambda^{r} \mathcal{E}\right) .
$$

Remark 3.9. - 1) One has $C_{2}(\overline{\mathcal{E}}, r, a) \ll \overline{\mathcal{E}, r} a$.

2) When $\overline{\mathcal{E}}$ is a direct sum of Hermitian line bundles, the term $\varrho\left(\Lambda^{r} \overline{\mathcal{E}}^{\vee}, \bar{F}_{I}^{(r)}\right)$ vanishes. Hence we can choose $C_{2}$ to be

$$
\widehat{\mu}_{\max }\left(\Lambda^{r} \overline{\mathcal{E}}\right)+\frac{r}{2} \log \operatorname{rk}\left(S^{a} \mathcal{E}\right)+\frac{1}{2} \log \operatorname{rk}\left(\Lambda^{r} \mathcal{E}\right)+\log \sqrt{r !}+r \log a .
$$

3) If Bost's conjecture is true, then we can choose

$$
C_{2}=\widehat{\mu}_{\max }\left(\Lambda^{r} \overline{\mathcal{E}}\right)+\frac{1}{2} \log \operatorname{rk}\left(\Lambda^{r} \mathcal{E}\right)+\log \sqrt{r !}+r \log a .
$$

We apply Theorem 3.8 on $I_{X} \subset S^{\delta} \mathcal{E}$ and on $r=n-d$. Using the estimation (18), we obtain the following result:

Theorem 3.10. - Let $X \subset \mathbb{P}\left(\mathcal{E}_{K}\right)$ be a subvariety of dimension $d$ and of degree $\delta$. Denote by $\mathscr{X}$ the Zariski closure of $X$ in $\mathbb{P}(\mathcal{E})$. There exists a Hermitian vector subbundle $\bar{M}$ of $S^{(\delta-1)(n-d)} \overline{\mathcal{E}}$ satisfying

$$
\widehat{\mu}_{\min }(\bar{M}) \geqslant-(n-d) h_{\mathrm{Ph}}(X)-C_{3}
$$

and such that the subscheme of $\mathbb{P}(\mathcal{E})$ defined by vanishing of $M$ contains the singular loci of fibres of $\mathscr{X}$ but not the generic point of $\mathscr{X}$, where the constant $C_{3}=C_{3}(\overline{\mathcal{E}}, d, \delta)$ is defined as

$$
(n-d) C_{1}(\overline{\mathcal{E}}, d, \delta)+C_{2}(\overline{\mathcal{E}}, n-d, \delta) .
$$

Furthermore, one has $C_{3}(\overline{\mathcal{E}}, d, \delta) \ll \overline{\mathcal{E}, d} \delta$.

Proof. - We take $\bar{M}=\bar{I}_{X}^{(n-d)}$, where $\bar{I}_{X}$ is defined in page 14. Let $\tilde{X}$ be the subvariety of $\mathbb{P}\left(\mathcal{E}_{K}\right)$ defined by vanishing of $I_{X, K}$ and $\widetilde{\mathscr{X}}$ be its Zariski closure in $\mathbb{P}(\mathcal{E})$ (which is defined by vanishing of $I_{X}$ since $I_{X}$ is saturated). By Jacobian criterion, the subscheme of $\mathbb{P}(\mathcal{E})$ defined by vanishing of $M$ coincides with the locus of singular points of fibres of $\widetilde{\mathscr{X}}$, which contains the locus of singular points of fibres of $\mathscr{X}$. The inequality (28) is a consequence of (24) and (20). The last assertion results from Remarks 3.7 and 3.91 ). 


\section{Estimations of Hilbert-Samuel functions}

In this section, we discuss the estimations of the geometric and arithmetic HilbertSamuel functions. We fix in this section a Hermitian vector bundle $\overline{\mathcal{E}}$ of rank $n+1$ over Spec $\mathcal{O}_{K}$ and a subvariety $X \subset \mathbb{P}\left(\mathcal{E}_{K}\right)$ which is of dimension $d \geqslant 1$ and of degree $\delta$. Denote by $\mathscr{X}$ the Zariski closure of $X$ in $\mathbb{P}(\mathcal{E})$. Let $\mathcal{L}=\mathcal{O}_{\mathbb{P}(\mathcal{E})}(1)$ be the universal line bundle. We equip it with the Fubini-Study metrics and then obtain a Hermitian line bundle $\overline{\mathcal{L}}$ on $\mathbb{P}(\mathcal{E})$. For any integer $D \geqslant 1$, let $E_{D}:=H^{0}\left(\mathbb{P}(\mathcal{E}), \mathcal{L}^{\otimes D}\right)$ and $r(D)$ be its rank; let $F_{D}$ be the saturation of the image of $E_{D}$ in $H^{0}\left(\mathscr{X},\left.\mathcal{L}\right|_{\mathscr{X}} ^{\otimes D}\right)$ by the homomorphism of restriction of sections and let $r_{1}(D):=\operatorname{rk} F_{D}$.

4.1. Estimations of the geometric Hilbert-Samuel function. - In this section, we recall several known results on explicit estimations of the geometric HilbertSamuel function. Let $X \subset \mathbb{P}\left(\mathcal{E}_{K}\right)$ be a closed subvariety of dimension $d$ and of degree $\delta$. The (geometric) Hilbert-Samuel function of $X$ is by definition the function on $\mathbb{N}_{*}$ which sends $D \in \mathbb{N}_{*}$ to the rank of $H^{0}\left(X,\left.\mathcal{L}\right|_{X} ^{\otimes D}\right)$. By the asymptotic Riemann-Roch theorem, one has the following relation

$$
\operatorname{rk} H^{0}\left(X,\left.\mathcal{L}\right|_{X} ^{\otimes D}\right)=\frac{\delta}{d !} D^{d}+O\left(D^{d-1}\right) .
$$

However, here our concern is to obtain the upper and lower bounds of this quantity which hold for any $D$ in $\mathbb{N}_{*}$ except an explicit finite subset. In this direction there is a result of Kollár and Matsusaka [33] which asserts that when $X$ is normal, one has

$$
\left|\operatorname{rk} H^{0}\left(X,\left.\mathcal{L}\right|_{X} ^{\otimes D}\right)-\frac{\delta}{d !} D^{d}+\frac{\left(\left.K_{X} \cdot \mathcal{L}\right|_{X} ^{d-1}\right)}{2(d-1) !} D^{d-1}\right| \leqslant C \cdot D^{d-2},
$$

where $C$ is an explicitly computable constant depending only on $\delta$ and $\left(\left.K_{X} \cdot \mathcal{L}\right|_{X} ^{d-1}\right)$, $K_{X}$ being the dualizing line bundle of $X$. However, here we need the estimations independent of the dualizing sheaf (but asymptotically less precise than the estimation of Kollár and Matsusaka). For the upper bound, we refer to the following result of Chardin [18] (see also 43, 价).

Proposition 4.1. - For any integer $D \geqslant 1$, one has

$$
\operatorname{rk} H^{0}\left(X,\left.\mathcal{L}\right|_{X} ^{\otimes D}\right) \leqslant \delta\left(\begin{array}{c}
D+d-1 \\
d
\end{array}\right)+\left(\begin{array}{c}
D+d-1 \\
d-1
\end{array}\right) .
$$

The proof relies on the generic hyperplane intersection of $X$ and proceed by induction on the dimension $d$. For details see [1, §1.2].

Remark 4.2. - Chardin has actually proved the following upper bound for the function $r_{1}(D)$ :

$$
\forall D \geqslant 1, \quad r_{1}(D)=\operatorname{rk} F_{D} \leqslant \delta\left(\begin{array}{c}
D+d \\
d
\end{array}\right)
$$

As for the lower bound of $\operatorname{rk} H^{0}\left(X,\left.\mathcal{L}\right|_{X} ^{\otimes D}\right)$, the following is an elementary result, which can be found in the book of Kollár 34. 
Proposition 4.3. - For any integer $D \geqslant \delta$, one has

$$
\operatorname{rk} H^{0}\left(X,\left.\mathcal{L}\right|_{X} ^{\otimes D}\right) \geqslant \frac{\delta}{d !}(D+1-\delta)^{d}
$$

The proof consists of projecting generically $X$ to a hypersurface of degree $\delta$, which we refer to [34, p. 92].

In [43], Sombra has proved the following (optimal) lower bound for the function $r_{1}(D)$, which holds for all $D$.

Proposition 4.4. - For any integer $D \geqslant 1$, one has

$$
r_{1}(D) \geqslant\left(\begin{array}{c}
D+d+1 \\
d+1
\end{array}\right)-\left(\begin{array}{c}
D-\delta+d+1 \\
d+1
\end{array}\right) .
$$

4.2. Lower bound of the arithmetic Hilbert-Samuel function. - In this subsection, we reformulate a result of David and Philippon 22 on an explicit lower bound of the arithmetic Hilbert-Samuel function in the framework of the slope method. Note that their argument relies on the higher Chow forms introduced by Philippon [37. We begin by a reminder on it.

Let $m \geqslant 1$ be an integer. Denote by $W_{m}$ the product variety $\mathbb{P}\left(\mathcal{E}_{K}\right) \times$ $\mathbb{P}\left(S^{m}\left(\mathcal{E}_{K}\right)^{\vee}\right)^{d+1}$. Let $\Gamma_{m} \subset W_{m}$ be the incidence subvariety classifying all points $\left(\alpha, u_{0}, \cdots, u_{d}\right)$ such that $\alpha^{\otimes m}\left(u_{0}\right)=\cdots \alpha^{\otimes m}\left(u_{d}\right)=0$, where we have considered a quotient of rank one of $S^{m}\left(\mathcal{E}_{K}\right)^{\vee}$ as a subspace of rank one of $S^{m}\left(\mathcal{E}_{K}\right)$. Denote by $p_{m}: W_{m} \rightarrow \mathbb{P}\left(\mathcal{E}_{K}\right)$ and $q_{m}: W_{m} \rightarrow \mathbb{P}\left(S^{m}\left(\mathcal{E}_{K}^{\vee}\right)\right)^{d+1}$ the two projections.

The following proposition asserts the existence of the higher Chow forms, which generalizes Proposition 3.1. This result has also been proved in [37]. See [7] for a geometric proof.

Proposition 4.5. - Let $X \subset \mathbb{P}\left(\mathcal{E}_{K}\right)$ be a subvariety. Then the set-theoretical intersection $\Gamma_{m} \cap p_{m}^{-1}(X)$ is irreducible. Furthermore, if we consider $\Gamma_{m} \cap p_{m}^{-1}(X)$ as a reduced subvariety of $W$, then the scheme-theoretical image $q_{m}\left(\Gamma_{m} \cap p_{m}^{-1}(X)\right)$ is a hypersurface of multi-degree $\left(\delta m^{d}, \cdots, \delta m^{d}\right)$.

Denote by $\bar{\Phi}_{X}^{[m]}$ the Hermitian line subbundle of $S^{\delta m^{d}}\left(S^{m}(\overline{\mathcal{E}})^{\vee}\right)^{\otimes(d+1)}$ corresponding to the hypersurface in Proposition 4.5. By definition, one has $\bar{\Phi}_{X}=\bar{\Phi}_{X}^{[1]}$.

We recall a result in [7] (Theorem 4.3.2) where the Arakelov degree of higher Chow form $\bar{\Phi}_{X}^{[m]}$ is computed by using the Philippon height.

Proposition 4.6. - The following equality holds:

$$
\widehat{\mu}\left(\bar{\Phi}_{X}^{[m]}\right)=m^{d+1} \widehat{\mu}\left(\bar{\Phi}_{X}\right) .
$$

In order to obtain an effective estimation of the arithmetic Hilbert-Samuel function, we need the following algebraic construction of $\Phi_{X}^{[m]}$ given by Philippon in [37].

Denote by $A$ the symmetric algebra $\operatorname{Sym}_{\mathcal{O}_{K}}(\mathcal{E})$. The algebra $A$ identifies with $\bigoplus_{D \geqslant 0} H^{0}\left(\mathbb{P}(\mathcal{E}), \mathcal{L}^{\otimes D}\right)$. For any integer $m \geqslant 1$, define

$$
\mathcal{O}_{K}^{[m]}:=\operatorname{Sym}_{\mathcal{O}_{K}}\left(S^{m}(\mathcal{E})^{\vee \oplus(d+1)}\right)
$$




$$
A^{[m]}:=\operatorname{Sym}_{\mathcal{O}_{K}}\left(\mathcal{E} \oplus S^{m}(\mathcal{E})^{\vee \oplus(d+1)}\right) \cong \operatorname{Sym}_{\mathcal{O}_{K}^{[m]}}\left(\mathcal{O}_{K}^{[m]} \otimes \mathcal{O}_{K} \mathcal{E}\right) .
$$

As a symmetric algebra, the $\mathcal{O}_{K}$-algebra $\mathcal{O}_{K}^{[m]}$ is naturally graded. We equip $A^{[m]}$ with the grading which is induced from that of $A$, or equivalently the natural grading corresponding to the symmetric $\mathcal{O}_{K}^{[m]}$-algebra structure of $\operatorname{Sym}_{\mathcal{O}_{K}^{[m]}}\left(\mathcal{O}_{K}^{[m]} \otimes_{\mathcal{O}_{K}} \mathcal{E}\right)$.

For $i \in\{0,1, \cdots, d\}$, let $\operatorname{tr}_{i}$ be the image of the trace element of $S^{m}(\mathcal{E}) \otimes S^{m}(\mathcal{E})^{\vee}$ in $A^{[m]}$ via the $(i+1)^{\mathrm{th}}$ component of $S^{m}(\mathcal{E})^{\mathrm{v} \oplus(d+1)}$. It is a homogeneous element of degree $m$ in $A^{[m]}$. Recall that the trace element corresponds to Id $: S^{m}(\mathcal{E}) \rightarrow S^{m}(\mathcal{E})$ through the natural isomorphism $S^{m}(\mathcal{E}) \otimes S^{m}(\mathcal{E})^{\vee} \cong \operatorname{Hom}_{\mathcal{O}_{K}}\left(S^{m}(\mathcal{E}), S^{m}(\mathcal{E})\right.$ ). Let $\mathfrak{I}$ be the kernel of the restriction homomorphism $A \rightarrow \bigoplus_{D \geqslant 0} H^{0}\left(\mathscr{X}, \mathcal{L}^{\otimes D}\right)$. It is a homogeneous ideal of $A$. Denote by

$$
\mathfrak{I}^{[m]}=A^{[m]} \mathfrak{I}+A^{[m]} \operatorname{tr}_{0}+\cdots+A^{[m]} \operatorname{tr}_{d} .
$$

It is a homogeneous ideal of $A^{[m]}$.

Proposition 4.7. - 1) The ideal

$$
\mathfrak{C}^{[m]} \mathfrak{I}:=\bigcup_{D \geqslant 0}\left(\mathfrak{I}^{[m]}:_{\mathcal{O}_{K}^{[m]}} A_{D}\right)
$$

of $\mathcal{O}_{K}^{[m]}$ is principal, and is generated by $\Phi_{X}^{[m]}$.

2) Assume that $D \geqslant(n-d)(\delta-1)+1$. Then for any integer $m \geqslant 1$, one has

$$
\left(\mathfrak{I}^{[m]}:_{\mathcal{O}_{K}^{[m]}} A_{D+m(d+1)-d}\right) \neq 0 .
$$

See [37, Proposition 1.5] for the proof of 1), [22, Proposition 4.2] for that of 2), see also loc. cit. page 528 .

By using Proposition 4.7, we obtain the following lower bound of the arithmetic Hilbert-Samuel function, which reformulates [22, Proposition 4.10] in the language of the slope theory.

Theorem 4.8. - Let $X \subset \mathbb{P}\left(\mathcal{E}_{K}\right)$ be a closed subvariety of dimension d and of degree $\delta, \mathscr{X}$ be the Zariski closure of $X$ in $\mathbb{P}(\mathcal{E})$. For any integer $D \geqslant 1$ let $\bar{A}_{D}=S^{D} \overline{\mathcal{E}}$ and let $\mathfrak{I}_{D}$ be the kernel of the restriction homomorphism $A_{D} \rightarrow H^{0}\left(\mathscr{X}, \mathcal{L}^{\otimes D}\right)$. Then, for any integer $D>(n-d)(\delta-1)+1$, one has

$$
\begin{aligned}
\widehat{\mu}\left(\overline{A_{D} / \mathfrak{I}_{D}}\right) \geqslant- & \frac{(D-(n-d)(\delta-1)-2)^{d+1}}{(d+1)^{d+1} \operatorname{rk}\left(A_{D} / \mathfrak{I}_{D}\right)}\left[\widehat{\mu}\left(\bar{\Phi}_{X}\right)+\delta(d+1) \widehat{\mu}_{\min }(\overline{\mathcal{E}})\right] \\
& -\frac{1}{2} D \log (n+1)+D \widehat{\mu}_{\text {min }}(\overline{\mathcal{E}})-\frac{\varrho^{\left(D \operatorname{rk}\left(A_{D} / \mathfrak{I}_{D}\right)\right)}\left(\overline{\mathcal{E}}^{\vee}\right)}{\operatorname{rk}\left(A_{D} / \mathfrak{I}_{D}\right)} .
\end{aligned}
$$

Proof. - Let $m \in \mathbb{N}_{*}$ be a parameter which will be chosen in the end of the proof. Let $\mathcal{O}_{K}^{[m]}, A^{[m]}$ and $\mathfrak{I}^{[m]}$ be as in (33), (34) and (35) respectively. For any integer $D \geqslant 1, A_{D}^{[m]}$ is a free $\mathcal{O}_{K}^{[m]}$-module of rank $\left(\begin{array}{c}D+n \\ n\end{array}\right)$. The $D^{\text {th }}$ homogeneous component $\mathfrak{I}_{D}^{[m]}$ of $\mathfrak{I}^{[m]}$ can be considered as a sub- $\mathcal{O}_{K}^{[m]}$-module of $A_{D}^{[m]}$. By definition, one has

$$
\mathfrak{I}_{D}^{[m]}=\mathcal{O}_{K}^{[m]} \mathfrak{I}_{D} \oplus \mathcal{O}_{K}^{[m]} A_{D-m} \operatorname{tr}_{0} \oplus \cdots \oplus \mathcal{O}_{K}^{[m]} A_{D-m} \operatorname{tr}_{d} .
$$


Consider $M_{D}^{[m]}:=\Lambda_{\mathcal{O}_{K}^{[n]}}^{\left(\begin{array}{c}n+n \\ n\end{array}\right)} \mathfrak{I}_{D}^{[m]}$. It is a sub- $\mathcal{O}_{K}^{[m]}$-module of

$$
\Lambda_{\mathcal{O}_{K}^{(m]}}^{\left(\begin{array}{c}
n+n \\
n
\end{array}\right)} A_{D}^{[m]} \cong \mathcal{O}_{K}^{[m]} \otimes_{\mathcal{O}_{K}} \operatorname{det}\left(A_{D}\right)
$$

so it determines an ideal of $\mathcal{O}_{K}^{[m]}$ by twisting the module by $\operatorname{det}\left(A_{D}\right)^{\vee}$. Note that here $\operatorname{det}\left(A_{D}\right)$ is defined as $\Lambda_{\mathcal{O}_{K}}^{\mathrm{rk}\left(A_{D}\right)} A_{D}$. Let $I_{D}^{[m]}=\mathfrak{I}_{D} \oplus A_{D-m} \operatorname{tr}_{0} \oplus \cdots \oplus A_{D-m} \operatorname{tr}_{d}$. One has $M_{D}^{[m]}=\mathcal{O}_{K}^{[m]} \otimes_{\mathcal{O}_{K}} \Lambda_{\mathcal{O}_{K}}^{\left(D^{n} n\right.} I_{D}^{[m]}$. By proposition 4.72$)$, if $D \geqslant(n-d)(\delta-1)+1+$ $m(d+1)-d$ (which we always assume in the rest of the proof), then $M_{D}^{[m]} \neq 0$, and hence the canonical image of

$$
\operatorname{det} \mathfrak{I}_{D} \otimes \Lambda_{\mathcal{O}_{K}}^{\mathrm{rk}\left(A_{D} / \mathfrak{I}_{D}\right)}\left(A_{D-m} \operatorname{tr}_{0} \oplus \cdots \oplus A_{D-m} \operatorname{tr}_{d}\right)
$$

in $\Lambda_{\mathcal{O}_{K}}^{\mathrm{rk} A_{D}} I_{D}^{[m]}$ is non-zero. By definition of the Chow form, the canonical homomorphism

$\operatorname{det}\left(A_{D}\right)^{\vee} \otimes \operatorname{det}\left(\mathfrak{I}_{D}\right) \otimes \Lambda_{\mathcal{O}_{K}}^{\mathrm{rk}\left(A_{D} / I_{D}\right)}\left(A_{D-m} \operatorname{tr}_{0} \oplus \cdots \oplus A_{D-m} \operatorname{tr}_{d}\right) \longrightarrow S^{\mathrm{rk}\left(A_{D} / \mathfrak{I}_{D}\right)}\left(S^{m}(\mathcal{E})^{\vee}\right)$ factories through

$$
\Phi_{X}^{[m]} \otimes \bigoplus_{i_{0}+\cdots+i_{d}=r_{D, m}} \bigotimes_{j=0}^{d} S^{m}(\mathcal{E})^{\vee \otimes i_{j}},
$$

where $r_{D, m}=\operatorname{rk}\left(A_{D} / \mathfrak{I}_{D}\right)-\delta(d+1) m^{d}$. Hence the slope inequality implies

$$
\begin{aligned}
& -\operatorname{rk}\left(A_{D} / \mathfrak{I}_{D}\right) \cdot \widehat{\mu}\left(\overline{A_{D} / \mathfrak{I}_{D}}\right)+\widehat{\mu}_{\min }\left(\Lambda_{\mathcal{O}_{K}}^{\mathrm{rk}\left(A_{D} / \mathfrak{I}_{D}\right)}\left(\bar{A}_{D-m}^{\oplus(d+1)}\right)\right) \\
\leqslant & \widehat{\mu}\left(\bar{\Phi}_{X}^{[m]}\right)+\max _{i_{0}+\cdots+i_{d}=r_{D, m}} \widehat{\mu}_{\max }\left(\bigotimes_{j=0}^{d} S^{m}(\mathcal{E})^{\vee \otimes i_{j}}\right),
\end{aligned}
$$

where $A_{D}$ is equipped with symmetric product metrics.

By Proposition 2.1 2) and Lemma A.1 4), one obtains

$$
\begin{aligned}
& \widehat{\mu}_{\min }\left(\Lambda_{\mathcal{O}_{K}}^{\mathrm{rk}\left(A_{D} / \mathfrak{I}_{D}\right)}\left(\bar{A}_{D-m}^{\oplus(d+1)}\right)\right) \\
\geqslant & \widehat{\mu}_{\min }\left(\left(\bar{A}_{D-m}^{\oplus(d+1)}\right)^{\otimes \operatorname{rk}\left(A_{D} / \mathfrak{I}_{D}\right)}\right)-\frac{1}{2} \operatorname{rk}\left(A_{D} / \mathfrak{I}_{D}\right) \log \left(\operatorname{rk}\left(A_{D} / \mathfrak{I}_{D}\right)\right) \\
= & \widehat{\mu}_{\min }\left(\bar{A}_{D-m}^{\otimes \operatorname{rk}\left(A_{D} / \mathfrak{I}_{D}\right)}\right)-\frac{1}{2} \operatorname{rk}\left(A_{D} / \mathfrak{I}_{D}\right) \log \left(\operatorname{rk}\left(A_{D} / \mathfrak{I}_{D}\right)\right) .
\end{aligned}
$$

Note that $\bar{A}_{D-m}$ is a quotient of $\overline{\mathcal{E}}^{\otimes D-m}$. Hence

$$
\begin{aligned}
& \widehat{\mu}_{\min }\left(\bar{A}_{D-m}^{\otimes \operatorname{rk}\left(A_{D} / \mathfrak{I}_{D}\right)}\right) \geqslant \widehat{\mu}_{\min }\left(\overline{\mathcal{E}}^{\otimes(D-m) \operatorname{rk}\left(A_{D} / \mathfrak{I}_{D}\right)}\right) \\
\geqslant & \operatorname{rk}\left(A_{D} / \mathfrak{I}_{D}\right)(D-m) \widehat{\mu}_{\min }(\overline{\mathcal{E}})-\varrho^{\left((D-m) \operatorname{rk}\left(A_{D} / \mathfrak{I}_{D}\right)\right)}\left(\overline{\mathcal{E}}^{\vee}\right) .
\end{aligned}
$$

Furthermore, if $i_{0}, \cdots, i_{d}$ are positive integers such that $i_{0}+\cdots+i_{d}=r_{D, m}$, then

$$
\begin{aligned}
& \widehat{\mu}_{\max }\left(\bigotimes_{j=0}^{d} S^{m}(\overline{\mathcal{E}})^{\vee \otimes i_{j}}\right)=-\widehat{\mu}_{\min }\left(\bigotimes_{j=0}^{d} S^{m}(\overline{\mathcal{E}})^{\otimes i_{j}}\right) \\
\leqslant & -\widehat{\mu}_{\min }\left(\mathcal{E}^{\otimes m r_{D, m}}\right) \leqslant-m r_{D, m} \widehat{\mu}_{\min }(\overline{\mathcal{E}})+\varrho^{\left(m r_{D, m}\right)}\left(\overline{\mathcal{E}}^{\vee}\right),
\end{aligned}
$$


where we reminder that $r_{D, m}:=\operatorname{rk}\left(A_{D} / \mathfrak{I}_{D}\right)-\delta(d+1) m^{d}$.

Combining the inequalities $(38),(39),(40)$ and $(41)$, we obtain

$$
\begin{aligned}
\widehat{\mu}\left(\overline{A_{D} / \mathfrak{I}_{D}}\right) \geqslant- & \frac{\widehat{\mu}\left(\bar{\Phi}_{X}^{[m]}\right)}{\operatorname{rk}\left(A_{D} / \mathfrak{I}_{D}\right)}-\frac{1}{2} \log \left(\operatorname{rk}\left(A_{D} / \mathfrak{I}_{D}\right)\right)+D \widehat{\mu}_{\min }(\overline{\mathcal{E}}) \\
& -\delta(d+1) \frac{m^{d+1}}{\operatorname{rk}\left(A_{D} / \mathfrak{I}_{D}\right)} \widehat{\mu}_{\min }(\overline{\mathcal{E}})-\frac{\varrho^{\left(D \operatorname{rk}\left(A_{D} / \mathfrak{I}_{D}\right)\right)}\left(\overline{\mathcal{E}}^{\vee}\right)}{\operatorname{rk}\left(A_{D} / \mathfrak{I}_{D}\right)},
\end{aligned}
$$

where we have applied (4) and used the fact that $\left(\varrho^{(n)}\left(\overline{\mathcal{E}}^{\vee}\right)\right)_{n \geqslant 1}$ is an increasing sequence. Since $\widehat{\mu}\left(\bar{\Phi}_{X}^{[m]}\right)=m^{d+1} \widehat{\mu}\left(\bar{\Phi}_{X}\right)$ and since $\operatorname{rk}\left(A_{D} / \mathfrak{I}_{D}\right) \leqslant \operatorname{rk}\left(A_{D}\right) \leqslant(n+1)^{D}$, we obtain

$$
\begin{aligned}
\widehat{\mu}\left(\overline{A_{D} / \mathfrak{I}_{D}}\right) \geqslant- & \frac{m^{d+1}}{\operatorname{rk}\left(A_{D} / \mathfrak{I}_{D}\right)}\left[\widehat{\mu}\left(\bar{\Phi}_{X}\right)+\delta(d+1) \widehat{\mu}_{\min }(\overline{\mathcal{E}})\right] \\
& -\frac{1}{2} D \log (n+1)+D \widehat{\mu}_{\min }(\overline{\mathcal{E}})-\frac{\varrho^{\left(D \operatorname{rk}\left(A_{D} / \mathfrak{I}_{D}\right)\right)}\left(\overline{\mathcal{E}}^{\vee}\right)}{\operatorname{rk}\left(A_{D} / \mathfrak{I}_{D}\right)} .
\end{aligned}
$$

Since the inequality (42) is verified for any $m$ satisfying $D \geqslant(n-d)(\delta-1)+1+$ $m(d+1)-d$. Thus we obtain the theorem by taking

$$
m=\left\lfloor\frac{D-(n-d)(\delta-1)-1+d}{d+1}\right\rfloor \geqslant \frac{D-(n-d)(\delta-1)-2}{d+1} .
$$

Remark 4.9. - As an $\mathcal{O}_{K}$-module, $A_{D}$ is isomorphic to $E_{D}$. However, the symmetric product metrics on $A_{D}$ differ from those of $\bar{E}_{D}$ (see $\$ 2.3$, notably Remark 2.6). Thus, if we equip $F_{D}$ with quotient metric of those of $\bar{E}_{D}$, then for any integer $D>(n-d)(\delta-1)+1$, one has

$$
\begin{gathered}
\widehat{\mu}\left(\bar{F}_{D}\right) \geqslant \frac{(D-(n-d)(\delta-1)-2)^{d+1}}{\operatorname{rk}\left(F_{D}\right)(d+1)^{d+1}}\left[h_{\mathrm{Ph}}(X)-\delta(d+1) \widehat{\mu}_{\min }(\overline{\mathcal{E}})\right] \\
-D \log (n+1)+D \widehat{\mu}_{\min }(\overline{\mathcal{E}})-\frac{\varrho^{\left(D \operatorname{rk}\left(A_{D} / \mathfrak{I}_{D}\right)\right)}\left(\overline{\mathcal{E}}^{\vee}\right)}{\operatorname{rk}\left(A_{D} / \mathfrak{I}_{D}\right)},
\end{gathered}
$$

where we have used the estimation $\operatorname{rk}\left(E_{D}\right) \leqslant(n+1)^{D}$. Recall the following explicit estimations of the rank of $F_{D}$ by functions on $D, \delta$, and $d$ (see Remark 4.2):

$$
\operatorname{rk}\left(F_{D}\right) \leqslant \delta\left(\begin{array}{c}
D+d \\
d
\end{array}\right) \leqslant \frac{\delta}{d !}(D+d)^{d} .
$$

Combining (44), we obtain that the following inquality holds for any integer $D$ such that $D \geqslant(n-d)(\delta-1)+2$.

$$
\frac{1}{D} \widehat{\mu}\left(\bar{F}_{D}\right) \geqslant C_{4}(D, d, \delta)\left[h_{\mathrm{Ph}}(X)-\delta(d+1) \widehat{\mu}_{\min }(\overline{\mathcal{E}})\right]-C_{5}(n, \overline{\mathcal{E}}),
$$


where the constants $C_{4}$ and $C_{5}$ are defined as

$$
\begin{gathered}
C_{4}(D, d, \delta)=\frac{d !}{\delta(d+1)^{d+1}}\left(\frac{D-(n-d)(\delta-1)-2}{D+d}\right)^{d+1}, \\
C_{5}(n, \overline{\mathcal{E}})=\log (n+1)-\widehat{\mu}_{\min }(\overline{\mathcal{E}})-\frac{\varrho^{\left(D \operatorname{rk}\left(A_{D} / \mathfrak{I}_{D}\right)\right)}\left(\overline{\mathcal{E}}^{\vee}\right)}{D \operatorname{rk}\left(A_{D} / \mathfrak{I}_{D}\right)} .
\end{gathered}
$$

Note that $C_{4}(D, d, \delta) \geqslant d ! / \delta(2 d+2)^{d+1}$ once $D \geqslant 2(n-d)(\delta-1)+d+4$.

4.3. Upper bound of the arithmetic Hilbert-Samuel function. - We show that a variant of Proposition 2.1 permits to obtain an upper bound of the (normalized) arithmetic Hilbert-Samuel function $\widehat{\mu}\left(\bar{F}_{D}\right)$. We actually find an explicit upper bound of $\widehat{\mu}_{\max }\left(\bar{F}_{D}\right)$ which holds for any $D \geqslant 1$. Let us begin by a reminder on the essential minimum.

Denote by $\bar{K}$ an algebraic closure of $K$. Let $X$ be a subvariety of $\mathbb{P}\left(\mathcal{E}_{K}\right)$. The essential minimum of $X$ (relatively to the Hermitian line bundle $\overline{\mathcal{L}}$ ) is by definition

$$
\widehat{\mu}_{\text {ess }}(X):=\sup _{\substack{\emptyset \neq U \subset X \\ U \text { open in } X}} \inf _{P \in U(\bar{K})} h_{\overline{\mathcal{L}}}(P) .
$$

By [45, Lemma 6.5], the essential minimum $\widehat{\mu}_{\text {ess }}(X)$ is finite, and one has the following estimation

$$
\widehat{\mu}_{\mathrm{ess}}(X) \leqslant \frac{h_{\overline{\mathcal{L}}}(X)}{\delta},
$$

where $h_{\overline{\mathcal{L}}}(X)$ is the Arakelov height of $X$ with respect to $\overline{\mathcal{L}}$, and $\delta$ is the degree of $X$.

Theorem 4.10. - For any integer $D \geqslant 1$, one has

$$
\widehat{\mu}_{\max }\left(\bar{F}_{D}\right) \leqslant D \widehat{\mu}_{\text {ess }}(X)+\frac{1}{2} \log r_{1}(D) .
$$

Proof. - Let $t$ be a real number such that $t>\widehat{\mu}_{\text {ess }}(X)$. Denote by $\mathcal{B}_{t}$ the class of algebraic points $P$ of $X$ such that $h_{\overline{\mathcal{L}}}(P) \leqslant t$. Let $\varphi_{D}$ be the evaluation map

$$
F_{D, \bar{K}} \longrightarrow \bigoplus_{P \in \mathcal{B}_{t}} P^{*} \mathcal{L}_{\bar{K}}^{\otimes D}
$$

By definition, the family $\mathcal{B}_{t}$ is Zariski dense in $X$, so $\varphi_{D}$ is injective. Therefore, there exists a subset $\mathcal{B}_{t, D}$ of $\mathcal{B}_{t}$ such that the composition homomorphism $\psi_{D}:=\operatorname{pr}_{\mathcal{B}_{t, D}} \circ \varphi_{D}$ is an isomorphism, where

$$
\operatorname{pr}_{\mathcal{B}_{t, D}}: \bigoplus_{P \in \mathcal{B}} P^{*} \mathcal{L}_{\bar{K}}^{\otimes D} \rightarrow \bigoplus_{P \in \mathcal{B}_{t, D}} P^{*} \mathcal{L} \frac{\otimes D}{K}
$$

is the canonical projection. The slope inequality (see Proposition 2.1) implies that

$$
\widehat{\mu}_{\max }\left(\bar{F}_{D}\right) \leqslant D t+h\left(\psi_{D}\right) .
$$

Note that $h\left(\psi_{D}\right) \leqslant \frac{1}{2} \log r_{1}(D)$. Since $t>\widehat{\mu}_{\text {ess }}(X)$ is arbitrary, we obtain the assertion. 
Remark 4.11. - The inequality (49), combined with the estimations (48) and the trivial estimation $r_{1}(D) \leqslant(n+1)^{D}$, gives an explicit upper bound for $\widehat{\mu}_{\max }\left(\bar{F}_{D}\right)$ in terms of the degree, the dimension and the Arakelov height of $X$ :

$$
\forall D \geqslant 1, \quad \widehat{\mu}_{\max }\left(\bar{F}_{D}\right) \leqslant \frac{h_{\overline{\mathcal{L}}}(X)}{\delta} D+\frac{D}{2} \log (n+1) .
$$

By the comparison of the Arakelov height and the Philippon height (see for example [7, Theorem 4.3.8]), on obtains

$$
\forall D \geqslant 1, \quad \widehat{\mu}_{\max }\left(\bar{F}_{D}\right) \leqslant \frac{h_{\mathrm{Ph}}(X)}{\delta} D+D \log (n+1) .
$$

\section{Appendix A}

\section{Computation of norms of linear operators}

In this subsection, we compute the norms of several operators acting on several tensor powers of a Hermitian vector space. These computations have been useful in the application of the slope inequalities, notably in the estimation of the heights of $K$-linear homomorphisms.

Lemma A.1. - Let $m \in \mathbb{N}$ and $V$ be a Hermitian space of dimension $m$.

1) The canonical homomorphism $\alpha: V \otimes V^{\vee} \rightarrow \mathbb{C}$ has norm $\sqrt{m}$.

2) For any $d \in\{0, \cdots, m-1\}$, denote by $\beta_{d}: V \otimes V^{\otimes(d+1)} \otimes V^{\vee} \rightarrow \Lambda^{d+1} V$ the homomorphism which sends $x \otimes y_{0} \otimes \cdots \otimes y_{d} \otimes \xi$ to

$$
\xi(x) y_{0} \wedge \cdots \wedge y_{d}-\sum_{i=0}^{d} \xi\left(y_{i}\right) y_{0} \wedge \cdots \wedge y_{i-1} \wedge x \wedge y_{i+1} \wedge \cdots \wedge y_{d}
$$

Then the norm of $\beta_{d}$ is $\sqrt{(d+2) !(m-d-1)}$.

3) For any integer a such that $a \geqslant 1$, denote by $D_{a}: S^{a} V \rightarrow V \otimes S^{a-1} V$ the homomorphism which sends $v_{1} \cdots v_{a}$ to

$$
\sum_{i=1}^{a} v_{i} \otimes\left(v_{1} \cdots v_{i-1} v_{i+1} \cdots v_{a}\right)
$$

Then the norm of $D_{a}$ is $a$.

4) Let $r$ be an integer such that $1 \leqslant r \leqslant m$. Denote by $\gamma_{r}: V^{\otimes r} \rightarrow \Lambda^{r} V$ the canonical homomorphism. The the norm of $\gamma_{r}$ is $\sqrt{r !}$.

Proof. - Let $\left(e_{i}\right)_{i=1}^{m}$ be an orthonormal basis of $V$ and let $\left(e_{i}^{\vee}\right)_{i=1}^{m}$ be its dual basis, which is an orthonormal basis of $V^{\vee}$.

1) The homomorphism $\alpha$ sends $\sum_{1 \leqslant i, j \leqslant m} \lambda_{i j} e_{i} \otimes e_{j}^{\vee}$ to $\sum_{i=1}^{m} \lambda_{i i}$. Hence $\|\alpha\|=\sqrt{m}$. 
2) Note that $\beta_{d}$ sends $\sum_{i, \underline{j}, k} \lambda_{i, \underline{j}, k} e_{i} \otimes e_{j_{0}} \otimes \cdots \otimes e_{j_{d}} \otimes e_{k}^{\vee}$ to

$$
\sum_{i, \underline{j}, k} \lambda_{i, \underline{j}, k}\left(\delta_{i k} e_{j_{0}} \wedge \cdots \wedge e_{j_{d}}-\sum_{a=0}^{d} \delta_{j_{a} k} e_{j_{0}} \wedge \cdots \wedge e_{j_{a-1}} \wedge e_{i} \wedge e_{j_{a+1}} \wedge \cdots \wedge e_{j_{d}}\right),
$$

where $\underline{j}$ stands for $\left(j_{0}, \cdots, j_{d}\right)$, and $\delta_{\alpha \beta}=1$ if $\alpha=\beta$ and $\delta_{\alpha \beta}=0$ else. Let $u_{0}, \cdots, u_{d}$ be integers such that $1 \leqslant u_{0}<\cdots<u_{d} \leqslant m$ and $\underline{u}=\left(u_{0}, \cdots, u_{d}\right)$. The symmetric group $\mathfrak{S}_{d+1}$ acts on $\{1, \cdots, m\}^{d+1}$ by permuting the components. In other words, $\sigma \in \mathfrak{S}_{d+1}$ sends $\left(v_{0}, \cdots, v_{d}\right)$ to $\left(v_{\sigma(0)}, \cdots, v_{\sigma(d)}\right)$. Denote by sgn : $\mathfrak{S}_{d+1} \rightarrow\{ \pm 1\}$ the sign function. If we write (51) as a linear combination in the basis $\left(e_{v_{0}} \wedge \cdots \wedge e_{v_{d}}\right)_{1 \leqslant v_{0}<\cdots<v_{d} \leqslant m}$, then the coefficient of $e_{u_{0}} \wedge \cdots \wedge e_{u_{d}}$ is

$$
\begin{aligned}
& \sum_{\sigma \in \mathfrak{S}_{d+1}} \sum_{i=1}^{m} \operatorname{sgn}(\sigma) \lambda_{i, \sigma(\underline{u}), i}-\sum_{a=0}^{d} \sum_{\sigma \in \mathfrak{S}_{d+1}} \sum_{k=1}^{m} \operatorname{sgn}(\sigma) \lambda_{u_{\sigma(a)}, \sigma^{(a, k)}(\underline{u}), k} \\
= & \sum_{\sigma \in \mathfrak{S}_{d+1}} \sum_{\substack{1 \leqslant i \leqslant m \\
i \neq u_{\sigma(0)}, \cdots, u_{\sigma(d)}}} \operatorname{sgn}(\sigma) \lambda_{i, \sigma(\underline{u}), i}-\sum_{a=0}^{d} \sum_{\sigma \in \mathfrak{S}_{d+1}} \sum_{\substack{1 \leqslant k \leqslant m \\
k \neq u_{\sigma(a)}}} \operatorname{sgn}(\sigma) \lambda_{u_{\sigma(a)}, \sigma^{(a, k)}(\underline{u}), k},
\end{aligned}
$$

where $\sigma^{(a, k)}(\underline{u})=\left(u_{\sigma(0)}, \cdots, u_{\sigma(a-1)}, k, u_{\sigma(a+1)}, \cdots, u_{(\sigma(d))}\right)$. If $a$ and $b$ are two integers such that $0 \leqslant a \neq b \leqslant d$, and if $\sigma \in \mathfrak{S}_{d+1}$, we denote by $\sigma_{a, b} \in \mathfrak{S}_{d+1}$ such that $\sigma_{a, b}(c)=\sigma(c)$ for any $c \in\{0, \cdots, d\} \backslash\{a, b\}$ and that $\sigma_{a, b}(a)=\sigma(b)$, $\sigma_{a, b}(b)=\sigma(a)$. Note that, with this notation, the equality

$$
\lambda_{u_{\sigma(a)}, \sigma^{(a, k)}(\underline{u}), k}=\lambda_{u_{\sigma_{a, b}(b)}, \sigma_{a, b}^{(b, k)}(\underline{u}), k}
$$

holds provided that $k=u_{\sigma(b)}$. Moreover, one has $\operatorname{sgn}(\sigma)=-\operatorname{sgn}\left(\sigma_{a, b}\right)$. Therefore, the formula (52) may be simplified as

$$
\sum_{\sigma \in \mathfrak{S}_{d+1}} \sum_{\substack{1 \leqslant i \leqslant m \\ i \neq u_{\sigma(0)}, \cdots, u_{\sigma(d)}}} \operatorname{sgn}(\sigma) \lambda_{i, \sigma(\underline{u}), i}-\sum_{a=0}^{d} \sum_{\sigma \in \mathfrak{S}_{d+1}} \sum_{\substack{1 \leqslant k \leqslant m \\ k \neq u_{\sigma(0)}, \cdots, u_{\sigma(d)}}} \operatorname{sgn}(\sigma) \lambda_{u_{\sigma(a)}, \sigma^{(a, k)}(\underline{u}), k} .
$$

Hence the norm of $\beta_{d}$ is equal to $\sqrt{(d+2) !(m-d-1)}$.

3) For any $J=\left(J_{l}\right)_{l=1}^{m} \in \mathbb{N}^{m}$, let $|J|=J_{1}+\cdots+J_{m}, J !=J_{1} ! \cdots J_{m}$ ! and $e^{J}=e_{1}^{J_{1}} \cdots e_{m}^{J_{m}} \in S^{|J|} H$. Then $\left(e^{J}\right)_{|J|=a}$ is an orthogonal base of $S^{a} V$. Note that the norm of $e^{J}$ is $\sqrt{J ! / a !}$. For any integer $l=1, \cdots, m$, let $\alpha_{l}$ be the element in $\mathbb{N}^{m}$ whose $l^{\text {th }}$ coordinate is 1 and whose other coordinates are zero. If $x=\sum_{\left|J^{\prime}\right|=a} \lambda_{J^{\prime}} e^{J^{\prime}}$ is an element of $S^{a} V$, the homomorphism $D_{a}$ sends $x$ to

$$
\sum_{\left|J^{\prime}\right|=a} \lambda_{J^{\prime}} \sum_{l=1}^{m} J_{l}^{\prime} e_{l} \otimes e^{J^{\prime}-\alpha^{(l)}}=\sum_{|J|=a-1} \sum_{l=1}^{m}\left(J_{l}+1\right) \lambda_{J+\alpha(l)} e_{l} \otimes e^{J},
$$


where we have used the convention $e^{J}=0$ if $J \notin \mathbb{N}^{m}$. Therefore

$$
\begin{aligned}
\left\|D_{a}(x)\right\|^{2} & =\sum_{|J|=a-1} \sum_{l=1}^{m}\left(J_{l}+1\right)^{2} \lambda_{J+\alpha^{(l)}}^{2} \frac{J !}{(a-1) !}=\sum_{|J|=a-1} \sum_{l=1}^{m}\left(J_{l}+1\right) \lambda_{J+\alpha^{(l)}}^{2} \frac{\left(J+\alpha^{(l)}\right) !}{(a-1) !} \\
& =a \sum_{\left|J^{\prime}\right|=a} \lambda_{J^{\prime}}^{2} \frac{J^{\prime} !}{a !} \sum_{l=1}^{m} J_{l}^{\prime}=a^{2}\|x\|^{2} .
\end{aligned}
$$

Hence the norm of $D_{a}$ is $a$.

4) The homomorphism $\gamma_{r}$ sends $\sum_{\underline{i}} \lambda_{\underline{i}} e_{i_{1}} \otimes \cdots \otimes e_{i_{r}}$ to $\sum_{\underline{i}} \lambda_{\underline{i}} e_{i_{1}} \wedge \cdots \wedge e_{i_{r}}$, where $\underline{i}=$ $\left(i_{1}, \cdots, i_{r}\right) \in\{1, \cdots, m\}^{r}$. The symmetric group $\mathfrak{S}_{r}$ acts on $\{1, \cdots, m\}^{r}$ such that $\sigma \in \mathfrak{S}_{r}$ sends $\left(i_{1}, \cdots, i_{r}\right)$ to $\left(i_{\sigma(1)}, \cdots, i_{\sigma(r)}\right)$. With this notation, $\sum_{\underline{i}} \lambda_{\underline{i}} e_{i_{1}} \wedge \cdots \wedge e_{i_{r}}$ is simplified as

$$
\sum_{1 \leqslant i_{1}<\cdots<i_{r} \leqslant m}\left(\sum_{\sigma \in \mathfrak{S}_{r}} \operatorname{sgn}(\sigma) \lambda_{\sigma(\underline{i})}\right) e_{i_{1}} \wedge \cdots \wedge e_{i_{r}} .
$$

As $\left(e_{i_{1}} \wedge \cdots \wedge e_{i_{r}}\right)_{1 \leqslant i_{1}<\cdots<i_{r} \leqslant m}$ is an orthonormal basis of $\Lambda^{r} V$, we obtain that $\left\|\gamma_{r}\right\|=\sqrt{\# \mathfrak{S}_{r}}=\sqrt{r !}$

\section{References}

[1] D. Bertrand - Upper bounds for (geometric) Hilbert functions, in Introduction to algebraic independence theory, Lecture Notes in Math., vol. 1752, Springer, Berlin, 2001, p. $143-148$.

[2] E. Bombieri \& J. PilA - The number of integral points on arcs and ovals, Duke Mathematical Journal 59 (1989), no. 2, p. 337-357.

[3] J.-B. Bost - Périodes et isogénies des variétés abéliennes sur les corps de nombres (d'après D. Masser et G. Wüstholz), Astérisque (1996), no. 237, p. Exp. No. 795, 4, 115161, Séminaire Bourbaki, Vol. 1994/1995.

[4] _ Hermitian vector bundle and stability, Exposé à Oberwolfach, Conférence "Algebraische Zahlentheorie", le 24 juillet, 1997.

[5] _ Algebraic leaves of algebraic foliations over number fields, Publications Mathématiques. Institut de Hautes Études Scientifiques (2001), no. 93, p. 161-221.

[6] J.-B. Bost - Evaluation maps, slopes, and algebraicity criteria, in Proceedings of the International Congress of Mathematicians, Vol. II (Madrid, 2006) 537-562,, European Mathematical Society, 2007.

[7] J.-B. Bost, H. Gillet \& C. Soulé - Heights of projective varieties, Journal of the American Mathematical Society 7 (1994), no. 4, p. 903-1027.

[8] J.-B. Bost \& K. Künnemann - Hermitian vector bundles and extension groups on arithmetic schemes. I. geometry of numbers, prépublication http://arxiv.org/abs/math/ $0701343,2007$.

[9] N. Broberg - A note on a paper by R. Heath-Brown: "The density of rational points on curves and surfaces" [Ann. of Math. (2) 155 (2002), no. 2, 553-595], Journal für die Reine und Angewandte Mathematik 571 (2004), p. 159-178.

[10] W. D. Brownawell - Applications of Cayley-Chow forms, in Number theory (Ulm, 1987), Lecture Notes in Math., vol. 1380, Springer, New York, 1989, p. 1-18. 
[11] W. Brownawell - On the development of Gelfond's method., Number theory, Proc. Conf., Carbondale 1979, Lect. Notes Math. 751, 18-44 (1979)., 1979.

[12] T. D. Browning \& D. R. Heath-Brown - Counting rational points on hypersurfaces, Journal für die Reine und Angewandte Mathematik 584 (2005), p. 83-115.

[13] The density of rational points on non-singular hypersurfaces. I, The Bulletin of the London Mathematical Society 38 (2006), no. 3, p. 401-410.

[14] of the London Mathematical Society. Third Series 93 (2006), no. 2, p. 273-303, With an appendix by J. M. Starr.

[15] T. D. Browning, D. R. Heath-Brown \& P. Salberger - Counting rational points on algebraic varieties, Duke Mathematical Journal 132 (2006), no. 3, p. 545-578.

[16] F. CATANESE - Chow varieties, Hilbert schemes and moduli spaces of surfaces of general type, Journal of Algebraic Geometry 1 (1992), no. 4, p. 561-595.

[17] A. Chambert-Loir - Théorèmes d'algébricité en géométrie diophantienne (d'après J.B. Bost, Y. André, D. \& G. Chudnovsky), Astérisque (2002), no. 282, p. Exp. No. 886, viii, 175-209, Séminaire Bourbaki, Vol. 2000/2001.

[18] M. Chardin - Une majoration de la fonction de Hilbert et ses conséquences pour l'interpolation algébrique, Bulletin de la Société Mathématique de France 117 (1989), no. 3, p. $305-318$.

[19] H. CHEN - Explicit uniform estimation of rational points II. Hypersurface coverings, preprint, 2009.

[20] Maximal slope of tensor product of Hermitian vector bundles, Journal of Algebraic Geometry 18 (2009), no. 3, p. 575-603.

[21] W.-L. Chow \& B. L. VAN DER WAERDEN - Zur algebraischen Geometrie. IX, Mathematische Annalen 113 (1937), no. 1, p. 692-704.

[22] S. DAvid \& P. Philippon - Minorations des hauteurs normalisées des sous-variétés des tores, Annali della Scuola Normale Superiore di Pisa. Classe di Scienze. Serie IV 28 (1999), no. 3, p. 489-543.

[23] E. De Shalit \& O. Parzanchevski - On tensor products of semistable lattices, Prprint, 2006.

[24] J. Ellenberg \& A. VenkAtesh - On uniform bounds for rational points on nonrational curves, Int. Math. Res. Not. (2005), no. 35, p. 2163-2181.

[25] W. Fulton - Intersection theory, second ed., Ergebnisse der Mathematik und ihrer Grenzgebiete. 3. Folge. A Series of Modern Surveys in Mathematics [Results in Mathematics and Related Areas. 3rd Series. A Series of Modern Surveys in Mathematics], vol. 2, SpringerVerlag, Berlin, 1998.

[26] É. Gaudron - Pentes de fibrés vectoriels adéliques sur un corps globale, Rendiconti del Seminario Matematico della Università di Padova 119 (2008), p. 21-95.

[27] I. M. Gel'FAND, M. M. KAPRANOv \& A. V. Zelevinsky - Discriminants, resultants, and multidimensional determinants, Mathematics: Theory \& Applications, Birkhäuser Boston Inc., Boston, MA, 1994.

[28] A. GeLfond - Transcendental and algebraic numbers., New York: Dover Publications, Inc. VII, 190 p. , 1960 (English. Russian original).

[29] H. Gillet \& C. Soulé - An arithmetic Riemann-Roch theorem, Inventiones Mathematicae 110 (1992), no. 3, p. 473-543.

[30] P. Graftieaux - Formal groups and the isogeny theorem, Duke Mathematical Journal 106 (2000), no. 1, p. 81-121. 
[31] D. R. Heath-Brown - The density of rational points on curves and surfaces, Annals of Mathematics. Second Series 155 (2002), no. 2, p. 553-595.

[32] F. JoHn - Extremum problems with inequalities as subsidiary conditions, in Studies and Essays Presented to R. Courant on his 60th Birthday, January 8, 1948, Interscience Publishers, Inc., New York, N. Y., 1948, p. 187-204.

[33] J. Kollár \& T. Matsusaka - Riemann-Roch type inequalities, American Journal of Mathematics 105 (1983), no. 1, p. 229-252.

[34] J. Kollár - Lectures on resolution of singularities, Annals of Mathematics Studies, vol. 166, Princeton University Press, Princeton, NJ, 2007.

[35] M. LAurent - Sur quelques résultats récents de transcendance, Astérisque (1991), no. 198-200, p. 209-230 (1992), Journées Arithmétiques, 1989 (Luminy, 1989).

[36] J. V. NESTERENKo - Estimate of the orders of the zeroes of functions of a certain class, and their application in the theory of transcendental numbers, Izvestiya Akademii Nauk SSSR. Seriya Matematicheskaya 41 (1977), no. 2, p. 253-284, 477.

[37] P. Philippon - Critères pour l'indépendance algébrique, Institut des Hautes Études Scientifiques. Publications Mathématiques (1986), no. 64, p. 5-52.

[38] Sur des hauteurs alternatives. II, Université de Grenoble. Annales de l'Institut Fourier 44 (1994), no. 4, p. 1043-1065.

[39] J. Pila - Density of integral and rational points on varieties, Astérisque (1995), no. 228, p. 4, 183-187, Columbia University Number Theory Seminar (New York, 1992).

[40] H. RANDRIAmbololona - Hauteurs pour les sous-schémas et exemples d'utilisation de méthodes arakeloviennes en théorie de l'approximation diophantienne, Thèse de l'Université Paris XI, UFR Scientifique d'Orsay, Janvier 2002.

[41] P. SAlberger - On the density of rational and integral points on algebraic varieties, Journal für die Reine und Angewandte Mathematik 606 (2007), p. 123-147.

[42] _ Rational points of bounded height on projective surfaces, Mathematische Zeitschrift 258 (2008), p. 805-826.

[43] M. SombrA - Bounds for the Hilbert function of polynomial ideals and for the degrees in the Nullstellensatz, Journal of Pure and Applied Algebra 117/118 (1997), p. 565-599, Algorithms for algebra (Eindhoven, 1996).

[44] C. Soulé - Géométrie d'Arakelov et théorie des nombres transcendants, Astérisque (1991), no. 198-200, p. 355-371 (1992), Journées Arithmétiques, 1989 (Luminy, 1989).

[45] S. ZhANG - Positive line bundles on arithmetic varieties, Journal of the American Mathematical Society 8 (1995), no. 1, p. 187-221.

June 17, 2009

Huay Chen, Université Paris Diderot - Paris 7, Institut de Mathématiques de Jussieu E-mail : chenhuayi@math.jussieu.fr 